\title{
Mathematical model with fractional order derivatives for Tuberculosis taking into account its relationship with HIV/AIDS and Diabetes
}

Erick Manuel Delgado Moya, Alain Pietrus, and Sergio Muniz Oliva

To cite this article:

E. M. D. Moya, A. Pietrus, and S. M. Oliva,"Mathematical model with fractional order derivatives for

Tuberculosis taking into account its relationship with HIV/AIDS and Diabetes", Jambura J. Biomath, vol. 2, no. 2 , pp. $80-95,2021$

DOI: https://doi.org/10.34312/jjbm.v2i2.11553

(C) 2021 Author(s).

Articles You may be interested in

Analisis kestabilan dan kontrol optimal model matematika penyebaran penyakit Ebola dengan variabel kontrol berupa karantina

E. A. S. Megananda, C. Alfiniyah, and M. Miswanto http://dx.doi.org/10.34312/jjbm.v2i1.10258

Parameters estimation of generalized Richards model for COVID-19 cases in Indonesia using genetic algorithm

M. Rayungsari, M. Aufin, and N. Imamah

http://dx.doi.org/10.34312/jjbm.v1i1.6910

Model Matematika Penyebaran COVID-19 dengan Penggunaan Masker Kesehatan dan Karantina

M. Manaqib, I. Fauziah, and E. Hartati http://dx.doi.org/10.34312/jjbm.v2i2.10483

Estimasi Reproduction Number Model Matematika Penyebaran Malaria di Sumba Tengah, Indonesia

E. M. Banni, M. A. Kleden, M. Lobo, and M. Z. Ndii

https://doi.org/10.34312/jjbm.v2i1.9971

Global stability of a fractional-order logistic growth model with infectious disease

H. S. Panigoro and E. Rahmi

https://doi.org/10.34312/jjbm.v1i2.8135 


\title{
Mathematical model with fractional order derivatives for Tuberculosis taking into account its relationship with HIV/AIDS and diabetes
}

\author{
Erick Manuel Delgado Moya ${ }^{1, *}$, Alain Pietrus $^{2}$, Sergio Muniz Oliva $^{3}$ \\ 1,3 Institute of Mathematics and Statistics, Department of Applied Mathematics, University of Sao Paulo, Brazil. \\ ${ }^{2}$ University of Antilles, Department of Mathematic and Computer Sciences, LAMIA (EA 4540), BP 250, 97159, \\ Pointe-a-Pitre, Guadeloupe, France \\ *Corresponding author. Email: erickmath@ime.usp.br
}

\begin{abstract}
In this paper, we present a mathematical model for the study of resistance to tuberculosis treatment using fractional derivatives in the Caputo sense. This model takes into account the relationship between Tuberculosis, HIV/AIDS, and diabetes and differentiates resistance cases into MDR-TB (multidrug-resistant tuberculosis) and XDR-TB (extensively drug-resistant tuberculosis). We present the basic results associated with the model and study the behavior of the disease-free equilibrium points in the different sub-populations, TB-Only, TB-HIV/AIDS, and TB-Diabetes. We performed computational simulations for different fractional orders ( $\alpha$-values) using an Adams-Bashforth-Moulton type predictor-corrector PECE method. Among the results obtained, we have that the MDR-TB cases in all sub-populations decrease at the beginning of the study for the different $\alpha$-values. In XDR-TB cases in the TB-Only sub-population, there is a decrease in the number of cases. XDR-TB cases in the TB-HIV/AIDS sub-population have differentiated behavior depending on $\alpha$. This knowledge helps to design an effective control strategy. The XDR-TB cases in diabetics increased throughout the study period and outperformed all resistant compartments for the different $\alpha$-values. We recommend special attention to the control of this compartment due to this growth.
\end{abstract}

Keywords: Caputo Fractional Derivatives; Diabetes; HIV/AIDS; Resistance; Tuberculosis

\section{Introduction}

Tuberculosis is a chronic bacterial infectious disease caused by "Mycobacterium Tuberculosis". The main medical problems faced is the efficacy of treatments for tuberculosis and extensively resistant tuberculosis (XDR-TB) [1]. The number of cases of tuberculosis in the world has been increasing annually.

The rifampicin, isoniazid, pyrazinamide, ethambutol and streptomycin belong to the first line for the treatment of tuberculosis, and amikacin, kanamycin, capreomycin, cycloserine, moxifloxacin, levofloxacin, ethionamide are in the second line of treatment. Multidrug-resistant tuberculosis (MDR-TB) is caused by Mycobacterium tuberculosis strains with resistance to at least isoniazid and rifampin. Extensively drug-resistant tuberculosis (XDR-TB) is defined as a strain resistant to any type of fluoroquinolones and at least one of the three following injectable drugs: amikacin, capreomycin, or kanamycin in addition to isoniazid and rifampicin.

Diabetes is a risk factor for lower respiratory infections including TB and is a significant factor for TB infections at the population level [2]. An important factor is that diabetes increases TB risk 1.5 to 7.8 times and the relative risk for TB among diabetes patients is 3.11 [3]. Diabetes can affect the effectiveness of first-line anti-TB drugs, particularly the use of rifampicin [4].

The rate of diabetes for HIV patients when they are infected is the same as for the general population. But certain metabolic factors related to HIV, and HIV therapy can increase the incidence of diabetes over time.

HIV has a great impact on the dissipation of tuberculosis, increasing the incidence and mortality rates of the disease. The use of alcohol, illicit drugs, smoking, the presence of diabetes influence the appearance of latent TB and can interfere with the results of TB-HIV / AIDS co-infection (cure, death, and abandonment of the treatment) [5].

Two classic problems that have motivated the use of fractional analysis are the Tautochrone problem and the effect of memory in biological models [6]. Non-integer models have a memory effect, and most operators have 
crossover properties that improve predictions [7]. In particular, classical models with autonomous ordinary differential equations have no memory. Given an initial value, the solution is uniquely determined for any point in the domain. In general, this statement is not true for fractional differential equations [6]. One way to introduce the memory effect in mathematical models is to change the order of the derivative of a classical model so that it is not an integer [6, 8]. In particular, the authors of [9] study the effect of memory on epidemic evolution using fractional order differential equations. One type of memory used in ecology and epidemiology is hysteresis. This memory briefly consists in that the system depends on the current and previous conditions (influence of past events) [6, 10, 11]. For example, Pimenov et al. [12] incorporate hysteresis into a biological model.

In recent decades, the number of papers using fractional-order derivatives to model epidemics has increased [7, 13-21]. For example, for the study of Tuberculosis Ullah et al. [13] studied the dynamics of Tuberculosis with a fractional-order model specifically with Caputo derivative. Fatmawati et al. [7] studied a fractional model for the dynamics of tuberculosis dividing into two age groups using the fractional operators, Caputo and Atangana-Baleanu. Rosa and Torres [14] proposed a fractional-order mathematical model in Caputo sense for the transmission dynamics of tuberculosis and formulated a fractional optimal control problem. For TB and HIV / AIDS co-infection. Farman et al. [15] proposed a mathematical model of HIV / AIDS and TB co-infection using Caputo and Caputo-Fabrizo fractional derivative. Khan et al. [16] investigated the Mittag-Leffler fractional HIV-TB co-infection model and proved the existence of a unique set of the solutions of the model and the Hyers-Ulam stability. For the study of HIV/AIDS, Pinto and Carvalho [17] presented a fractional-order model for HIV infection, where latently infected cells, macrophages, and CTLs are included. Fatmawati et al. [18] presented a Caputo-derivative model for the spread of HIV / AIDS disease in a sex-divided population and for HIV and HCV (Hepatitis C virus) co-infection. Carvalho et al. [19] presented a fractional-order model for the $\mathrm{HIV} / \mathrm{HCV}$ co-infection dynamics. Concerning diabetes dynamics we have, Saleem et al. [20] presented a non-linear fractional-order model with Caputo-Fabrizio derivative for insulin therapy. Sakulrang et al. [21] proved that fractional-order differential equation models could give better fits than integer-order models concerning continuous glucose monitoring data from patients with type 1 diabetes. The objective of this paper is to study a mathematical model for resistance to treatment of Tuberculosis with the presence of HIV/AIDS and diabetes using fractional derivatives in the Caputo sense and to observe the behavior of resistance for different orders.

This paper is organized as follows: In Section 2 we present definitions and preliminary results. In Section 3 we introduce the model and the basic results then, we study the equilibrium points and their relationship with the basic reproduction number. Section 4 is devoted to numerical experimentation. We finish the paper with some conclusions in Section 5.

\section{Mathematical Preliminaries}

In this section, we present the fundamental definitions that are used in the document. We mention the definition of fractional derivative in the Caputo sense and a definition of the Mittag-Leffler function.

Definition 1. (See [22]) For $\alpha>0$, with $n-1<\alpha<n, n \in \mathbb{N}$, the fractional derivative in the sense of Caputo is defined as

$$
{ }^{c} \mathbb{D}_{t}^{\alpha} f(t)=\frac{d^{\alpha} f(t)}{d t^{\alpha}}:=\frac{1}{\Gamma(n-\alpha)} \int_{0}^{t}(t-s)^{(n-\alpha-1)} f^{(n)}(s) d s
$$

where $\Gamma($.$) is the gamma function. The Riemann-Liouville fractional integral is defined by$

$$
\mathbb{I}_{t}^{\alpha} f(t)=\frac{1}{\Gamma(\alpha)} \int_{0}^{t}(t-s)^{(\alpha-1)} f(s) d s
$$

Note: We have for any $p \in \mathbb{N},|z| \rightarrow \infty$ and $\frac{\alpha \pi}{2}<|\arg (z)| \leq \pi$, that $[23,24]$

$$
\mathbb{E}_{\alpha, \beta}(z)=-\sum_{k=1}^{w} \frac{z^{-k}}{\Gamma(\beta-z \alpha)}+O\left(|z|^{-1-w}\right) .
$$

where $\mathbb{E}_{\alpha, \beta}$ is two-parameters Mittag-Leffler function $\alpha, \beta>0$.

\section{Model formulation}

In this section, we present the model with Caputo fractional derivative equations introduced in [25] and its basic properties. This model is used for the study of MDR-TB and XDR-TB, taking into account the influence of 


\section{HIV / AIDS and diabetes.}

The model has eighteen compartments that are: uninfected of $\mathrm{TB}, S_{T}, S_{H}$, $S_{D}$, latently infected, $E_{T}, E_{H}, E_{D}$, individuals infected and drug-sensitive TB $\left(I_{T_{1}}, I_{H_{1}}, I_{D_{1}}\right)$, infected MDR-TB $\left(I_{T_{2}}, I_{H_{2}}, I_{D_{2}}\right)$, infected XDR-TB $\left(R_{T_{1}}\right.$, $\left.R_{H_{1}}, R_{D_{1}}\right)$ and recovered of TB $\left(R_{T}, R_{H}, R_{D}\right)$ individuals where $T$ represent TB-Only, $H$ are HIV / AIDS cases and $D$ diabetics individuals.

The $M_{1}, M_{2}$ and $M_{3}$ are recruitment rates in the different sub-models, where we do not have HIV/AIDS or diabetes, HIV / AIDS and diabetes respectively. The rate of acquiring diabetes by use of antiretroviral treatment is $\alpha_{4}$ and the rate of an individual who becomes infected with HIV (sexual transmission or otherwise) and develops diabetes are $\alpha_{2}$ and $\alpha_{1}$ respectively.

The TB infection rate is defined as

$$
\lambda_{T}=\alpha^{*} \frac{I_{T_{1}}+I_{T_{2}}+R_{T_{1}}+\epsilon_{1}\left(I_{H_{1}}+I_{H_{2}}+R_{H_{1}}\right)+\epsilon_{2}\left(I_{D_{1}}+I_{D_{2}}+R_{D_{1}}\right)}{N}
$$

where $\alpha^{*}$ is the effective contact rate and $N$ is the total population. The parameters $\epsilon_{j}, j=1,2$ are modifications parameters, associated with increased infectivity in HIV/AIDS and diabetics patients. The natural death rate $\mu$ is the same from any compartment and $u_{1}$ and $u_{2}$ are death rate associated with HIV / AIDS and diabetes.

The $\eta$ is defined as the natural rate of progression of tuberculosis. The $\beta^{*}$ represents the proportion of active TB cases that are resistant. The $\omega_{1}, \omega_{2}$, are the parameters associated with the transmission of tuberculosis in the $\mathrm{HIV} / \mathrm{AIDS}$ and diabetes compartments where $\omega_{1}, \omega_{2}>1$.

We assume that TB-recovered $R_{i}, i=T, D, H$ acquire partial immunity so that from the recovered compartment (the cases that recover) enter the latent compartment with a parameter associated with TB reinfection and reactivation $\beta_{1}^{\prime}$ with $\beta_{1}^{\prime} \leq 1$. We define $\epsilon_{j}^{*}, j=1,2$ as the parameters modification associated with resistance to tuberculosis treatment in HIV / AIDS and diabetics.

The $t_{1}$ and $t_{2}$ are modifications of the parameters associated with diabetes or HIV infection in compartments where there is active tuberculosis infection. We define death from TB with a rate $d_{1}$, deaths from the combination TB and HIV / AIDS with a rate $d_{2}$ and deaths from the combination TB and diabetes with a rate $d_{3}$. We assume that $d_{3} \geq d_{1}$ and $d_{2} \geq d_{1}$ as diabetes and HIV/AIDS experience greater disease induced deaths than their corresponding only TB and we assume death from TB after the use of treatment. The rates $l_{1}, l_{2}$ and $l_{3}$ represent the cases that will be MDR-TB (first resistance). The expressions $\left(1-p_{1}\right) \eta,\left(1-p_{2}\right) \epsilon_{1}^{*} \eta$ and $\left(1-p_{3}\right) \epsilon_{2}^{*} \eta$ are the cases that in a first infection are going to be MDR-TB and $p_{1} \eta, p_{2} \epsilon_{1}^{*} \eta$ and $p_{3} \epsilon_{2}^{*} \eta$ are the cases that are going to be XDR-TB in a first infection. The $t_{3}$ parameter is associated with the combination of antirretroviral therapy and the treatment of tuberculosis and the possibility of developing diabetes. The $\eta_{11}, \eta_{12}$ and $\eta_{13}$ is the recovery rate after being sensitive TB and $m_{1}, m_{2}$ and $m_{3}$ is the recovery rate after being MDR-TB. The $t_{l}^{\prime}, l=1,2,3$ are modification parameters associated with TB deaths in MDR-TB cases.

The $\eta_{11}^{*}, \eta_{12}^{*}$ and $\eta_{13}^{*}$ are the recovery rate after being XDR-TB. The $t_{1}^{*}, t_{2}^{*}$ and $t_{3}^{*}$ are modification parameters associated with death by TB, death by combination TB-HIV / AIDS and by combination TB-Diabetes after being XDR-TB. We assume that $\eta_{1 l}>\eta_{1 l}^{*}$ and $m_{l}>\eta_{1 l}^{*}$ for $l=1,2,3$. The fractional derivative operator ${ }^{c} \mathbb{D}_{t}^{\alpha}$ has a dimension time ${ }^{-\alpha}$, then on the right-hand side of the model all parameters will have power dimension $\alpha$. The Table 1 shows the variables and parameters of the model.

Table 1. Variables and Parameters of model eq. (1)

\begin{tabular}{|c|c|c|c|}
\hline Parameter & Description & Parameter & Description \\
\hline$S_{T}, S_{H}, S_{D}$ & Uninfected of TB & $E_{T}, E_{H}, E_{D}$ & Latently infected \\
\hline$I_{T_{1}}, I_{H_{1}}, I_{D_{1}}$ & Drug-sensitive TB & $I_{T_{2}}, I_{H_{2}}, I_{D_{2}}$ & Infected MDR-TB \\
\hline$R_{T_{1}}, R_{H_{1}}, R_{D_{1}}$ & Infected XDR-TB & $R_{T}, R_{H}^{2}, R_{D}$ & Recovered of TB \\
\hline$M_{1}, M_{2}, M_{3}$ & Recruitment rates & $\alpha^{*}$ & Effective contact rates for TB infection \\
\hline$\alpha_{1}$ & Acquiring diabetes rate & $\alpha_{2}$ & Acquiring HIV rate \\
\hline$\alpha_{4}$ & Diabetes development rate by use of HIV therapy & $\omega_{1}, \omega_{2}, \epsilon_{1}, \epsilon_{2}$ & Modification parameters \\
\hline$\mu$ & Natural mortality rate & $\eta$ & Natural rate of progression to active TB \\
\hline$t_{1}, t_{2}, t_{3}, t_{1}^{*}, t_{2}^{*}, t_{3}^{*}$ & Modification parameters & $t_{1}^{\prime}, t_{2}^{\prime}, t_{3}^{\prime}$ & Modification parameters \\
\hline$\epsilon_{1}^{*}, \epsilon_{2}^{*}, \beta_{1}^{\prime}$ & Modification parameters & $l_{1}, l_{2}, l_{3}$ & Resistant TB development rates \\
\hline$d_{3}$ & TB-Diabetes induced death rate & $\mu_{1}, \mu_{2}$ & Death rate of HIV/AIDS and diabetes respectively. \\
\hline$m_{1}, m_{2}, m_{3}$ & $\mathrm{~TB}$ recovery rates for MDR-TB & $\beta^{*}$ & Proportion of active TB cases that are resistant. \\
\hline$\eta_{11}, \eta_{12}, \eta_{13}$ & TB recovery rates of drug-sensitive TB infected & $\eta_{14}, \eta_{15}, \eta_{16}$ & Resistant (XDR-TB) TB development rates after being MDR-TB \\
\hline$\eta_{11}^{*}, \eta_{12}^{*}, \eta_{13}^{*}$ & TB recovery rates of XDR-TB & $p_{1}, p_{2}, p_{3}$ & Rates related to developing XDR-TB resistance \\
\hline
\end{tabular}


The effectiveness of the TB treatment with the presence of HIV/AIDS and diabetes using Caputo's functional derivative can be expressed as:

$$
\begin{aligned}
& { }^{c} \mathbb{D}_{t}^{\alpha} S_{T}=M_{1}-\left(\mu+\alpha_{1}+\alpha_{2}+\lambda_{T}\right) S_{T} \text {, } \\
& { }^{c} \mathbb{D}_{t}^{\alpha} S_{H}=M_{2}+\alpha_{2}\left(S_{T}+S_{D}\right)-\left(\alpha_{4}+\mu+\mu_{1}+\omega_{1} \lambda_{T}\right) S_{H} \text {, } \\
& { }^{c} \mathbb{D}_{t}^{\alpha} S_{D}=M_{3}+\alpha_{4} S_{H}+\alpha_{1} S_{T}-\left(\alpha_{2}+\mu+\mu_{2}+\omega_{2} \lambda_{T}\right) S_{D} \text {, } \\
& { }^{c} \mathbb{D}_{t}^{\alpha} E_{T}=\lambda_{T}\left(S_{T}+\beta_{1}^{\prime} R_{T}\right)-\left(\alpha_{1}+\alpha_{2}+\mu+\eta\right) E_{T} \text {, } \\
& { }^{c} \mathbb{D}_{t}^{\alpha} E_{H}=\omega_{1} \lambda_{T}\left(S_{H}+\beta_{1}^{\prime} R_{H}\right)+\alpha_{2}\left(E_{T}+E_{D}\right)-\left(\epsilon_{1}^{*} \eta+\mu+\mu_{1}+\alpha_{4}\right) E_{H} \text {, } \\
& { }^{c} \mathbb{D}_{t}^{\alpha} E_{D}=\omega_{2} \lambda_{T}\left(S_{D}+\beta_{1}^{\prime} R_{D}\right)+\alpha_{4} E_{H}+\alpha_{1} E_{T}-\left(\alpha_{2}+\epsilon_{2}^{*} \eta+\mu+\mu_{2}\right) E_{D} \text {, } \\
& { }^{c} \mathbb{D}_{t}^{\alpha} I_{T_{1}}=\left(1-\beta^{*}\right) \eta E_{T}-\left(l_{1}+t_{1} \alpha_{1}+t_{2} \alpha_{2}+\mu+d_{1}+\eta_{11}\right) I_{T_{1}} \text {, } \\
& { }^{c} \mathbb{D}_{t}^{\alpha} I_{T_{2}}=\left(1-p_{1}\right) \beta^{*} \eta E_{T}+l_{1} I_{T_{1}}-\left(t_{1} \alpha_{1}+t_{2} \alpha_{2}+m_{1}+\mu+t_{1}^{\prime} d_{1}+\eta_{14}\right) I_{T_{2}} \text {, } \\
& { }^{c} \mathbb{D}_{t}^{\alpha} I_{H_{1}}=t_{2} \alpha_{2}\left(I_{T_{1}}+I_{D_{1}}\right)+\left(1-\beta^{*}\right) \epsilon_{1}^{*} \eta E_{H}-\left(l_{2}+\mu+\mu_{1}+d_{2}+\eta_{12}+t_{3} \alpha_{4}\right) I_{H_{1}} \text {, } \\
& { }^{c} \mathbb{D}_{t}^{\alpha} I_{H_{2}}=t_{2} \alpha_{2}\left(I_{T_{2}}+I_{D_{2}}\right)+\left(1-p_{2}\right) \epsilon_{1}^{*} \beta^{*} \eta E_{H}+l_{2} I_{H_{1}}-\left(m_{2}+\mu+\mu_{1}+t_{2}^{\prime} d_{2}+\eta_{15}+t_{3} \alpha_{4}\right) I_{H_{2}} \text {, } \\
& { }^{c} \mathbb{D}_{t}^{\alpha} I_{D_{1}}=t_{1} \alpha_{1} I_{T_{1}}+t_{3} \alpha_{4} I_{H_{1}}+\left(1-\beta^{*}\right) \epsilon_{2}^{*} \eta E_{D}-\left(l_{3}+t_{2} \alpha_{2}+\mu+\mu_{2}+d_{3}+\eta_{13}\right) I_{D_{1}} \text {, } \\
& { }^{c} \mathbb{D}_{t}^{\alpha} I_{D_{2}}=t_{1} \alpha_{1} I_{T_{2}}+t_{3} \alpha_{4} I_{H_{2}}+\left(1-p_{3}\right) \epsilon_{2}^{*} \beta^{*} \eta E_{D}+l_{3} I_{D_{1}}-\left(m_{3}+t_{2} \alpha_{2}+\mu+\mu_{2}+t_{3}^{\prime} d_{3}+\eta_{16}\right) I_{D_{2}} \text {, } \\
& { }^{c} \mathbb{D}_{t}^{\alpha} R_{T_{1}}=p_{1} \beta^{*} \eta E_{T}+\eta_{14} I_{T_{2}}-\left(\eta_{11}^{*}+t_{1} \alpha_{1}+t_{2} \alpha_{2}+\mu+t_{1}^{*} d_{1}\right) R_{T_{1}} \text {, } \\
& { }^{c} \mathbb{D}_{t}^{\alpha} R_{H_{1}}=p_{2} \beta^{*} \epsilon_{1}^{*} \eta E_{H}+\eta_{15} I_{H_{2}}+t_{2} \alpha_{2}\left(R_{T_{1}}+R_{D_{1}}\right)-\left(\eta_{12}^{*}+t_{3} \alpha_{4}+\mu+\mu_{1}+t_{2}^{*} d_{2}\right) R_{H_{1}} \text {, } \\
& { }^{c} \mathbb{D}_{t}^{\alpha} R_{D_{1}}=p_{3} \beta^{*} \epsilon_{2}^{*} \eta E_{D}+\eta_{16} I_{D_{2}}+t_{3} \alpha_{4} R_{H_{1}}+t_{1} \alpha_{1} R_{T_{1}}-\left(t_{2} \alpha_{2}+\eta_{13}^{*}+\mu+\mu_{2}+t_{3}^{*} d_{3}\right) R_{D_{1}} \text {, } \\
& { }^{c} \mathbb{D}_{t}^{\alpha} R_{T}=m_{1} I_{T_{2}}+\eta_{11} I_{T_{1}}+\eta_{11}^{*} R_{T_{1}}-\left(\alpha_{1}+\alpha_{2}+\mu+\beta_{1}^{\prime} \lambda_{T}\right) R_{T} \text {, } \\
& { }^{c} \mathbb{D}_{t}^{\alpha} R_{H}=m_{2} I_{H_{2}}+\eta_{12} I_{H_{1}}+\eta_{12}^{*} R_{H_{1}}+\alpha_{2}\left(R_{T}+R_{D}\right)-\left(\alpha_{4}+\mu+\mu_{1}+\beta_{1}^{\prime} \omega_{1} \lambda_{T}\right) R_{H} \text {, } \\
& { }^{c} \mathbb{D}_{t}^{\alpha} R_{D}=m_{3} I_{D_{2}}+\eta_{13} I_{D_{1}}+\eta_{13}^{*} R_{D_{1}}+\alpha_{1} R_{T}+\alpha_{4} R_{H}-\left(\alpha_{2}+\mu+\mu_{2}+\beta_{1}^{\prime} \omega_{2} \lambda_{T}\right) R_{D}
\end{aligned}
$$

with initial conditions:

$S_{T}(0)>0, S_{H}(0)>0, S_{D}(0)>0, E_{T}(0)>0, E_{H}(0)>0, E_{D}(0)>0, I_{T_{1}}(0)>0, I_{T_{2}}(0)>0, I_{H_{1}}(0)>0$, $I_{H_{2}}(0)>0, I_{D_{1}}(0)>0, I_{D_{2}}(0)>0, R_{T_{1}}(0)>0, R_{H_{1}}(0)>0, R_{D_{1}}(0)>0, R_{T}(0)>0, R_{H}(0)>0, R_{D}(0)>0$ and $\alpha \in(0,1]$.

\subsection{Basic properties of model}

Now, let us prove the existence and positivity of the solution of the system (1) and let us find the region where all variables are always nonnegative and the solutions remain positive with positive initial conditions, which we define as the biologically feasible region.

\section{Existence and non-negativity of solutions}

Let us consider

$$
\begin{aligned}
\Omega_{1}=\{x= & \left(S_{T}, S_{H}, S_{D}, E_{T}, E_{H}, E_{D}, I_{T_{1}}, I_{T_{2}}, I_{H_{1}}, I_{H_{2}}, I_{D_{1}}, I_{D_{2}}, R_{T_{1}}, R_{H_{1}}, R_{D_{1}}, R_{T}, R_{H}, R_{D}\right): \\
& \left.S_{T}, S_{H}, S_{D}, E_{T}, E_{H}, E_{D}, I_{T_{1}}, I_{T_{2}}, I_{H_{1}}, I_{H_{2}}, I_{D_{1}}, I_{D_{2}}, R_{T_{1}}, R_{H_{1}}, R_{D_{1}}, R_{T}, R_{H}, R_{D} \geq 0\right\}
\end{aligned}
$$

The following lemma and corollary will be used in the proof of Theorem 4 and they come from [26].

Lemma 2. (Generalized mean value theorem) Suppose that $f \in C[a, b]$ and ${ }^{c} \mathbb{D}_{t}^{\alpha} f \in C[a, b]$, for $\alpha \in(0,1]$. Then, $\forall t \in(a, b]$, with $a \leq \epsilon \leq t$ we have

$$
f(t)=f(a)+\frac{1}{\Gamma(\alpha)}{ }^{c} \mathbb{D}_{t}^{\alpha} f(\epsilon)(t-a)^{\alpha}
$$

where $\Gamma($.$) is the gamma function.$

Corollary 3. Consider that $f \in C[a, b]$ and ${ }^{c} \mathbb{D}_{t}^{\alpha} f \in C[a, b]$, for $\alpha \in(0,1]$. Then if

- ${ }^{c} \mathbb{D}_{t}^{\alpha} f(t) \geq 0, \forall t \in(a, b)$, then $f(t)$ is non-decreasing for each $t \in[a, b]$.

- ${ }^{c} \mathbb{D}_{t}^{\alpha} f(t) \leq 0, \forall t \in(a, b)$, then $f(t)$ is non-increasing for each $t \in[a, b]$.

Theorem 4. There is a unique solution $x(t)=\left(S_{T}, S_{H}, S_{D}, E_{T}, E_{H}, E_{D}, I_{T_{1}}, I_{T_{2}}, I_{H_{1}}, I_{H_{2}}, I_{D_{1}}, I_{D_{2}}, R_{T_{1}}, R_{H_{1}}, R_{D_{1}}\right.$, $\left.R_{T}, R_{H}, R_{D}\right)^{T}$ of the model (1) for $t \geq 0$ and the solution will remain in $\Omega_{1}$. 
proof. By Theorem 3.1 and Remark 3.2 of [27] we have that the solution in $(0, \infty)$ of the initial value problem (1) exists and is unique. Now, let's we will prove the positivity of the solution of the model (1). In order to do, we need to prove that for every hyperplane bounding the nonnegative orthant, the vector field points to $\Omega_{1}$. From model (1), we have:

$$
\begin{aligned}
& \left.{ }^{c} \mathbb{D}_{t}^{\alpha} S_{T}\right|_{S_{T}=0}=M_{1}>0, \\
& \left.{ }^{c} \mathbb{D}_{t}^{\alpha} S_{H}\right|_{S_{H}=0}=M_{2}>0 \text {, } \\
& \left.{ }^{c} \mathbb{D}_{t}^{\alpha} S_{D}\right|_{S_{D}=0}=M_{3}>0 \text {, } \\
& \left.{ }^{c} \mathbb{D}_{t}^{\alpha} E_{T}\right|_{E_{T}=0}=\lambda_{T}\left(S_{T}+\beta_{1}^{\prime} R_{T}\right) \geq 0, \\
& \left.{ }^{c} \mathbb{D}_{t}^{\alpha} E_{H}\right|_{E_{H}=0}=\omega_{1} \lambda_{T}\left(S_{H}+\beta_{1}^{\prime} R_{H}\right)+\alpha_{2}\left(E_{T}+E_{D}\right) \geq 0 \text {, } \\
& \left.{ }^{c} \mathbb{D}_{t}^{\alpha} E_{D}\right|_{E_{D}=0}=\omega_{2} \lambda_{T}\left(S_{D}+\beta_{1}^{\prime} R_{D}\right)+\alpha_{4} E_{H}+\alpha_{1} E_{T} \geq 0, \\
& \left.{ }^{c} \mathbb{D}_{t}^{\alpha} I_{T_{1}}\right|_{I_{T_{1}}=0}=\left(1-\beta^{*}\right) \eta E_{T} \geq 0 \text {, } \\
& \left.{ }^{c} \mathbb{D}_{t}^{\alpha} I_{T_{2}}\right|_{I_{T_{2}}=0}=\left(1-p_{1}\right) \beta^{*} \eta E_{T}+l_{1} I_{T_{1}} \geq 0 \text {, } \\
& \left.{ }^{c} \mathbb{D}_{t}^{\alpha} I_{H_{1}}\right|_{I_{H_{1}}=0}=t_{2} \alpha_{2}\left(I_{T_{1}}+I_{D_{1}}\right)+\left(1-\beta^{*}\right) \epsilon_{1}^{*} \eta E_{H} \geq 0 \text {, } \\
& \left.{ }^{c} \mathbb{D}_{t}^{\alpha} I_{H_{2}}\right|_{I_{H_{2}}=0}=t_{2} \alpha_{2}\left(I_{T_{2}}+I_{D_{2}}\right)+\left(1-p_{2}\right) \epsilon_{1}^{*} \beta^{*} \eta E_{H}+l_{2} I_{H_{1}} \geq 0, \\
& \left.{ }^{c} \mathbb{D}_{t}^{\alpha} I_{D_{1}}\right|_{I_{D_{1}}=0}=t_{1} \alpha_{1} I_{T_{1}}+t_{3} \alpha_{4} I_{H_{1}}+\left(1-\beta^{*}\right) \epsilon_{2}^{*} \eta E_{D} \geq 0, \\
& \left.{ }^{c} \mathbb{D}_{t}^{\alpha} I_{D_{2}}\right|_{I_{D_{2}}=0}=t_{1} \alpha_{1} I_{T_{2}}+t_{3} \alpha_{4} I_{H_{2}}+\left(1-p_{3}\right) \epsilon_{2}^{*} \beta^{*} \eta E_{D}+l_{3} I_{D_{1}} \geq 0 \text {, } \\
& \left.{ }^{c} \mathbb{D}_{t}^{\alpha} R_{T_{1}}\right|_{R_{T_{1}}=0}=p_{1} \beta^{*} \eta E_{T}+\eta_{14} I_{T_{2}} \geq 0, \\
& \left.{ }^{c} \mathbb{D}_{t}^{\alpha} R_{H_{1}}\right|_{R_{H_{1}}=0}=p_{2} \beta^{*} \epsilon_{1}^{*} \eta E_{H}+\eta_{15} I_{H_{2}}+t_{2} \alpha_{2}\left(R_{T_{1}}+R_{D_{1}}\right) \geq 0, \\
& \left.{ }^{c} \mathbb{D}_{t}^{\alpha} R_{D_{1}}\right|_{R_{D_{1}}=0}=p_{3} \beta^{*} \epsilon_{2}^{*} \eta E_{D}+\eta_{16} I_{D_{2}}+t_{3} \alpha_{4} R_{H_{1}}+t_{1} \alpha_{1} R_{T_{1}} \geq 0, \\
& \left.{ }^{c} \mathbb{D}_{t}^{\alpha} R_{T}\right|_{R_{T}=0}=m_{1} I_{T_{2}}+\eta_{11} I_{T_{1}}+\eta_{11}^{*} R_{T_{1}} \geq 0, \\
& \left.{ }^{c} \mathbb{D}_{t}^{\alpha} R_{H}\right|_{R_{H}=0}=m_{2} I_{H_{2}}+\eta_{12} I_{H_{1}}+\eta_{12}^{*} R_{H_{1}}+\alpha_{2}\left(R_{T}+R_{D}\right) \geq 0, \\
& \left.{ }^{c} \mathbb{D}_{t}^{\alpha} R_{D}\right|_{R_{D}=0}=m_{3} I_{D_{2}}+\eta_{13} I_{D_{1}}+\eta_{13}^{*} R_{D_{1}}+\alpha_{1} R_{T}+\alpha_{4} R_{H} \geq 0 .
\end{aligned}
$$

Using Corollary (3), we have that the solution will remain in $\Omega_{1}$.

\section{Biologically Feasible Region}

All the elements of the model (parameters and variables) are defined for a population of humans so they cannot be negative and we are going to show that $\Omega$ is positively invariant for the model (1).

Lemma 5. The closed set $\Omega=\left\{\left(S_{i}, E_{i}, I_{i_{1}}, I_{i_{2}}, R_{i_{1}}, R_{i}\right) \in \mathbb{R}_{+}^{18}, i=T, H, D: N(t) \leq \frac{M_{1}+M_{2}+M_{3}}{\mu}\right\}$ is positively invariant with respect to model (1).

proof. The derivative of the total population is

$$
\begin{aligned}
{ }^{c} \mathbb{D}_{t}^{\alpha} N(t)= & { }^{c} \mathbb{D}_{t}^{\alpha} S_{T}(t)+{ }^{c} \mathbb{D}_{t}^{\alpha} S_{H}(t)+{ }^{c} \mathbb{D}_{t}^{\alpha} S_{D}(t)+{ }^{c} \mathbb{D}_{t}^{\alpha} E_{T}(t)+{ }^{c} \mathbb{D}_{t}^{\alpha} E_{H}(t)+{ }^{c} \mathbb{D}_{t}^{\alpha} E_{D}(t)+ \\
& { }^{c} \mathbb{D}_{t}^{\alpha} I_{T_{1}}(t)+{ }^{c} \mathbb{D}_{t}^{\alpha} I_{T_{2}}(t)+{ }^{c} \mathbb{D}_{t}^{\alpha} I_{H_{1}}(t)+{ }^{c} \mathbb{D}_{t}^{\alpha} I_{H_{2}}(t)+{ }^{c} \mathbb{D}_{t}^{\alpha} I_{D_{1}}(t)+{ }^{c} \mathbb{D}_{t}^{\alpha} I_{D_{2}}(t)+ \\
& { }^{c} \mathbb{D}_{t}^{\alpha} R_{T_{1}}(t)+{ }^{c} \mathbb{D}_{t}^{\alpha} R_{H_{1}}(t)+{ }^{c} \mathbb{D}_{t}^{\alpha} R_{D_{1}}(t)+{ }^{c} \mathbb{D}_{t}^{\alpha} R_{T}(t)+{ }^{c} \mathbb{D}_{t}^{\alpha} R_{H}(t)+{ }^{c} \mathbb{D}_{t}^{\alpha} R_{D}(t)
\end{aligned}
$$

and we have

$$
{ }^{c} \mathbb{D}_{t}^{\alpha} N(t)+\mu N(t) \leq M_{1}+M_{2}+M_{3} .
$$

Applying the Laplace transform to (2), we have

$$
s^{\alpha} \phi(N)-s^{\alpha-1} \phi(0) \leq \frac{M_{1}+M_{2}+M_{3}}{s}-\mu \phi(N)
$$


which further gives

$$
\phi(N) \leq \frac{M_{1}+M_{2}+M_{3}}{s\left(s^{\alpha}+\mu\right)}+\frac{s^{\alpha-1}}{s^{\alpha}+\mu} N(0) .
$$

To continue the prove we use the following definitions.

Definition 6. The Laplace transform of the Caputo fractional derivatives of the function $\phi(t)$ with order $\alpha>0$ is defined as

$$
\mathcal{L}\left[{ }^{c} \mathbb{D}_{t}^{\alpha} \phi(t)\right]=s^{\alpha} \phi(s)-\sum_{v=0}^{n-1} \phi^{v}(0) s^{\alpha-v-1}
$$

Definition 7. The Laplace transform of the function $t^{\alpha_{1}-1} \mathbb{E}_{\alpha, \alpha_{1}}\left( \pm \lambda t^{\alpha}\right)$ is defined as

$$
\mathcal{L}\left[t^{\alpha_{1}-1} \mathbb{E}_{\alpha, \alpha_{1}}\left( \pm \lambda t^{\alpha}\right)\right]=\frac{s^{\alpha-\alpha_{1}}}{s^{\alpha} \mp \lambda}
$$

where $\mathbb{E}_{\alpha, \alpha_{1}}$ is two-parameters Mittag-Leffler function $\alpha, \alpha_{1}>0$. Futher, the Mittag-Leffler function satisfies the following equation [28]

$$
\mathbb{E}_{\alpha, \alpha_{1}}(f)=f \mathbb{E}_{\alpha, \alpha+\alpha_{1}}(f)+\frac{1}{\Gamma\left(\alpha_{1}\right)}
$$

Using the eqs. (5) to (7), we assumed that $\left(S_{T}(0), S_{H}(0), S_{D}(0), E_{T}(0), E_{H}(0), E_{D}(0), I_{T_{1}}(0), I_{T_{2}}(0)\right.$, $\left.I_{H_{1}}(0), I_{H_{2}}(0), I_{D_{1}}(0), I_{D_{2}}(0), R_{T_{1}}(0), R_{H_{1}}(0), R_{D_{1}}(0), R_{T}(0), R_{H}(0), R_{D}(0)\right) \in \mathbb{R}_{+}^{18}$, then

$$
N(t) \leq\left(M_{1}+M_{2}+M_{3}\right) t^{\alpha} \mathbb{E}_{\alpha, \alpha+1}\left(-\mu t^{\alpha}\right)+N(0) \mathbb{E}_{\alpha, 1}\left(-\mu t^{\alpha}\right) .
$$

Using the asymptotic behavior of the Mittag-Leffler function presented in the preliminaries, we can observed that $N(t) \rightarrow \frac{M_{1}+M_{2}+M_{3}}{\mu}$ as $t \rightarrow \infty$. The region $\Omega$ (biologically feasible region) is well established and all the solutions for the initial values that belong to $\Omega$ remain in $\Omega$ for each time $t>0$.

\subsection{Study of the equilibrium points and the basic reproduction number}

The basic reproduction number $\left(\Re_{0}\right)$ is among the most important quantities in infectious disease epidemiology. The $\Re_{0}$ is defined as the average number of an infection caused by a typical infected individual, in a population consisting only of susceptibles. If $0<\Re_{0}<1$ the infection will die out in the long run. But if $\Re_{0}>1$ the infection will be able to spread in a population. The higher the $\Re_{0}$ the more difficult it is to control the epidemic [29].

In this section, we study the equilibrium points and their relationship with the basic reproduction number. We base our study on the infection-free equilibrium points and the basic reproduction number is obtained using the new generation matrix theory [29]. We work on three sub-models, the sub-model where we do not have HIV / AIDS and diabetes (TB-Only), the sub-model where we have HIV/AIDS (TB-HIV/AIDS), and the submodel where we have diabetes (TB-Diabetes). The calculation of the basic reproduction numbers is in [25].

\section{TB-Only sub-model}

We have the TB-Only sub-model when $\left(S_{H}=S_{D}=E_{H}=E_{D}=I_{H_{1}}=I_{H_{2}}=I_{D_{1}}=I_{D_{2}}=R_{H_{1}}=R_{D_{1}}=R_{H}=\right.$ $R_{D}=0$ ) which is given by

$$
\begin{aligned}
{ }^{c} \mathbb{D}_{t}^{\alpha} S_{T} & =M_{1}-\left(\mu+\alpha_{1}+\alpha_{2}+\lambda_{T}\right) S_{T}, \\
{ }^{c} \mathbb{D}_{t}^{\alpha} E_{T} & =\lambda_{T}\left(S_{T}+\beta_{1}^{\prime} R_{T}\right)-\left(\alpha_{1}+\alpha_{2}+\eta+\mu\right) E_{T}, \\
{ }^{c} \mathbb{D}_{t}^{\alpha} I_{T_{1}} & =\left(1-\beta^{*}\right) \eta E_{T}-\left(l_{1}+t_{1} \alpha_{1}+t_{2} \alpha_{2}+\mu+d_{1}+\eta_{11}\right) I_{T_{1}} \\
{ }^{c} \mathbb{D}_{t}^{\alpha} I_{T_{2}} & =\left(1-p_{1}\right) \beta^{*} \eta E_{T}+l_{1} I_{T_{1}}-\left(m_{1}+\mu+t_{1}^{\prime} d_{1}+\eta_{14}+t_{1} \alpha_{1}+t_{2} \alpha_{2}\right) I_{T_{2}}, \\
{ }^{c} \mathbb{D}_{t}^{\alpha} R_{T_{1}} & =\beta^{*} p_{1} \eta E_{T}+\eta_{14} I_{T_{2}}-\left(\eta_{11}^{*}+\mu+t_{1}^{*} d_{1}+t_{1} \alpha_{1}+t_{2} \alpha_{2}\right) R_{T_{1}}, \\
{ }^{c} \mathbb{D}_{t}^{\alpha} R_{T} & =m_{1} I_{T_{2}}+\eta_{11} I_{T_{1}}+\eta_{11}^{*} R_{T_{1}}-\left(\mu+\beta_{1}^{\prime} \lambda_{T}+\alpha_{1}+\alpha_{2}\right) R_{T},
\end{aligned}
$$

with initial conditions:

$$
S_{T}(0)>0, E_{T}(0)>0, I_{T_{1}}(0)>0, I_{T_{2}}(0)>0, R_{T_{1}}(0)>0 \text {, and } R_{T}(0)>0 .
$$


The TB infection rate for this sub-model is defined as

$$
\lambda_{T}=\alpha^{*} \frac{I_{T_{1}}+I_{T_{2}}+R_{T_{1}}}{N_{T}}
$$

and the total population is given by

$$
N_{T}=S_{T}+E_{T}+I_{T_{1}}+I_{T_{2}}+R_{T_{1}}+R_{T}
$$

Due to biological constraints, the system (9) is studied in the following region:

$$
D_{1}=\left\{\left(S_{T}, E_{T}, I_{T_{1}}, I_{T_{2}}, R_{T_{1}}, R_{T}\right) \in \mathbb{R}_{+}^{6}: N_{T}(t) \leq \frac{M_{1}}{\mu}\right\}
$$

We can show for this sub-model (9) that the solutions, $\left(S_{T}(t), E_{T}(t), I_{T_{1}}(t), I_{T_{2}}(t), R_{T_{1}}(t), R_{T}(t)\right)$ are bounded and positively invariant in $D_{1}$ (biologically feasible region).

\section{Disease-free equilibrium point}

The disease-free equilibrium point of model (9) is given by $\epsilon_{0}^{T}=\left(S_{0}^{T}, 0,0,0,0,0\right)$, where $S_{0}^{T}=\frac{M_{1}}{\mu+\alpha_{1}+\alpha_{2}}$.

The basic reproduction number is calculated using next-generation matrix method in [25] and is defined as

$$
\Re_{0}^{T}=\frac{\alpha^{*} M_{1}\left(\left(1-\beta^{*}\right) \eta\left(k_{13} k_{14}+l_{1}\left(k_{14}+\eta_{14}\right)\right)+\left(1-p_{1}\right) \beta^{*} \eta k_{12}\left(k_{14}+\eta_{14}\right)+k_{12} k_{13} \beta^{*} \eta p_{1}\right)}{N_{T}\left(\alpha_{1}+\alpha_{2}+\mu\right) k_{11} k_{12} k_{13} k_{14}},
$$

where $k_{11}=\alpha_{1}+\alpha_{2}+\eta+\mu, k_{12}=l_{1}+t_{1} \alpha_{1}+t_{2} \alpha_{2}+\mu+d_{1}+\eta_{11}, k_{13}=\mu+t_{1}^{\prime} d_{1}+\eta_{14}+m_{1}+t_{1} \alpha_{1}+t_{2} \alpha_{2}$, and $k_{14}=\mu+t_{1}^{*} d_{1}+\eta_{11}^{*}+t_{1} \alpha_{1}+t_{2} \alpha_{2}$.

Lemma 8. (Theorem 2. of [30]) For any $q, r \in \mathbb{Z}_{+}$, such that $\operatorname{gcd}(q, r)=1$ Let $\alpha=\frac{q}{r}$ and we define $M=r$, then the disease-free equilibrium is locally asymptotically stable if $|\arg (\lambda)|>\frac{\pi}{2 M}$ for all roots $\lambda$ of the associated characteristic equation

$$
\operatorname{det}\left(\operatorname{diag}\left[\lambda^{q} \lambda^{q} \lambda^{q} \lambda^{q} \lambda^{q} \lambda^{q}-J\left(\epsilon_{0}^{T}\right)\right]\right)=0
$$

where $J\left(\epsilon_{0}^{T}\right)$ is the jacobian matrix of sub-model at $\epsilon_{0}^{T}$. The disease-free equilibrium $\epsilon_{0}^{T}$ is unstable if $\Re_{0}^{T}>1$.

proof. The Jacobian of sub-model at disease-free equilibrium is

$$
\left(\begin{array}{cccccc}
-\left(\mu+\alpha_{1}+\alpha_{2}\right) & 0 & 0 & 0 & 0 & 0 \\
0 & -k_{11} & 0 & 0 & 0 & 0 \\
0 & \left(1-\beta^{*}\right) \eta & -k_{12} & 0 & 0 & 0 \\
0 & \left(1-p_{1}\right) \beta^{*} \eta & l_{1} & -k_{13} & 0 & 0 \\
0 & p_{1} \beta^{*} \eta & 0 & \eta_{14} & -k_{14} & 0 \\
0 & 0 & \eta_{11} & m_{1} & \eta_{11}^{*} & -\left(\mu+\alpha_{1}+\alpha_{2}\right)
\end{array}\right)
$$

Expanding, $\operatorname{det}\left(\operatorname{diag}\left[\lambda^{q} I_{6}-J\left(\epsilon_{0}^{T}\right)\right]\right)=0$, where $I_{6}$ is the identity matrix of order 6 , we obtain the following equation in terms of $\lambda$ :

$$
\left(-\alpha_{1}-\alpha_{2}-\mu-\lambda^{q}\right)^{2}\left(\lambda^{4 q}+b_{1} \lambda^{3 q}+b_{2} \lambda^{2 q}+b_{3} \lambda^{q}+b_{4}\right)=0
$$

The arguments of the roots of equation $\left(-\alpha_{1}-\alpha_{2}-\mu-\lambda^{q}\right)=0$ are given by:

$$
\arg \left(\lambda_{k}\right)=\frac{\pi}{q}+k \frac{2 \pi}{q}>\frac{\pi}{M}>\frac{\pi}{2 M}
$$

where $k=0,1, \ldots,(q-1)$. Now, the coefficients $b_{i}{ }^{\prime}$ s of $p(\lambda)=\lambda^{4 q}+b_{1} \lambda^{3 q}+b_{2} \lambda^{2 q}+b_{3} \lambda^{q}+b_{4}$ are

$$
\begin{aligned}
& b_{1}=k_{11}+k_{12}+k_{13}+k_{14} \\
& b_{2}=k_{11} k_{12}+k_{11} k_{13}+k_{11} k_{14}+k_{12} k_{14}+k_{13} k_{14}-\frac{M_{1} \alpha^{*} \eta}{N_{T}\left(\mu+\alpha_{1}+\alpha_{2}\right)},
\end{aligned}
$$




$$
\begin{aligned}
b_{3}= & k_{11} k_{12} k_{13}+k_{11} k_{12} k_{14}+k_{11} k_{13} k_{14}+k_{12} k_{13} k_{14}-\frac{M_{1} \alpha^{*} \eta}{N_{T}\left(\mu+\alpha_{1}+\alpha_{2}\right)}\left(\left(1-\beta^{*}\right)\left(l_{1}+k_{13}\right)+\left(1-p_{1}\right) \beta^{*} \eta_{14}\right. \\
& +\beta^{*} p_{1}\left(\left(t_{1}^{\prime}-t_{1}^{*}\right) d_{1}+\left(m_{1}-\eta_{11}^{*}\right)+\eta_{14}\right), \\
b_{4}= & k_{11} k_{12} k_{13} k_{14}-\frac{M_{1} \alpha^{*} \eta}{N_{T}\left(\mu+\alpha_{1}+\alpha_{2}\right)}\left(\left(1-\beta^{*}\right)\left(k_{13} k_{14}+l_{1}\left(k_{14}+\eta_{14}\right)\right)+\left(1-p_{1}\right) k_{12} \beta^{*}\left(k_{14}+\eta_{14}\right)+k_{12} k_{13} \beta^{*} p_{1}\right) \\
= & \left(1-\Re_{0}^{T}\right) .
\end{aligned}
$$

The function $p(\lambda)$ has eigenvalues with negative real part if $b_{i}>0$ for $i=1,2,3,4$ and $b_{1} b_{2} b_{3}>b_{1}^{2} b_{4}+b_{3}^{2}$. All $b_{i}$ 's are greater than zero if $\Re_{0}^{T}<1$, and the conditions $b_{1} b_{2} b_{3}>b_{1}^{2} b_{4}+b_{3}^{2}$ ensure the stability of the disease-free case when $\Re_{0}^{T}<1$. If $\Re_{0}^{T}<1$, then the necessary condition fulfil for all the roots of characteristics equation i.e., $|\arg (\lambda)|>\frac{\pi}{2 M}$. Thus, the infection-free equilibrium point is locally asymptotically stable if $\Re_{0}^{T}<1$.

\subsection{TB-HIV/AIDS sub-model}

For the sub-model only with HIV/AIDS (TB-HIV/AIDS sub-model) $\left(S_{T}=S_{D}=E_{T}=E_{D}=I_{T_{1}}=I_{T_{2}}=I_{D_{1}}=\right.$ $I_{D_{2}}=R_{T_{1}}=R_{D_{1}}=R_{T}=R_{D}=0$ ) and is given by

$$
\begin{aligned}
& { }^{c} \mathbb{D}_{t}^{\alpha} S_{H}=M_{2}-\left(\alpha_{4}+\mu+\mu_{1}+\omega_{1} \lambda_{T}\right) S_{H}, \\
& { }^{c} \mathbb{D}_{t}^{\alpha} E_{H}=\omega_{1} \lambda_{T} S_{H}+\beta_{1}^{\prime} \omega_{1} \lambda_{T} R_{H}-\left(\epsilon_{1}^{*} \eta+\mu+\mu_{1}+\alpha_{4}\right) E_{H}, \\
& { }^{c} \mathbb{D}_{t}^{\alpha} I_{H_{1}}=\left(1-\beta^{*}\right) \epsilon_{1}^{*} \eta E_{H}-\left(l_{2}+\mu+\mu_{1}+d_{2}+\eta_{12}+t_{3} \alpha_{4}\right) I_{H_{1}}, \\
& { }^{c} \mathbb{D}_{t}^{\alpha} I_{H_{2}}=\left(1-p_{2}\right) \beta^{*} \epsilon_{1}^{*} \eta E_{H}+l_{2} I_{H_{1}}-\left(m_{2}+\mu+\mu_{1}+t_{2}^{\prime} d_{2}+\eta_{15}+t_{3} \alpha_{4}\right) I_{H_{2}}, \\
& { }^{c} \mathbb{D}_{t}^{\alpha} R_{H_{1}}=p_{2} \beta^{*} \epsilon_{1}^{*} \eta E_{H}+\eta_{15} I_{H_{2}}-\left(\eta_{12}^{*}+\mu+\mu_{1}+t_{2}^{*} d_{2}+t_{3} \alpha_{4}\right) R_{H_{1}}, \\
& { }^{c} \mathbb{D}_{t}^{\alpha} R_{H}=m_{2} I_{H_{2}}+\eta_{12} I_{H_{1}}+\eta_{12}^{*} R_{H_{1}}-\left(\mu+\mu_{1}+\beta_{1}^{\prime} \omega_{1} \lambda_{T}+\alpha_{4}\right) R_{H}
\end{aligned}
$$

with non-negative initial conditions and

$$
\lambda_{H}=\alpha^{*} \frac{\epsilon_{1}\left(I_{H_{1}}+I_{H_{2}}+R_{H_{1}}\right)}{N_{H}}
$$

where $N_{H}=S_{H}+E_{H}+I_{H_{1}}+I_{H_{2}}+R_{H_{1}}+R_{H}$.

Considering biological constraints, the system (13) will be studied in the following region:

$$
D_{2}=\left\{\left(S_{H}, E_{H}, I_{H_{1}}, I_{H_{2}}, R_{H_{1}}, R_{H}\right) \in \mathbb{R}_{+}^{6}: N_{H}(t) \leq \frac{M_{2}}{\mu}\right\} .
$$

It can be easily shown that te solution $\left(S_{H}(t), E_{H}(t), I_{H_{1}}(t), I_{H_{2}}(t), R_{H_{1}}(t), R_{H}(t)\right)$ of the system are bounded and positively invariant.

The disease-free equilibrium point is given by $\epsilon_{0}^{H}=\left(S_{0}^{H}, 0,0,0,0,0\right), \quad$ where $S_{0}^{H}=\frac{M_{2}}{\mu+\mu_{1}+\alpha_{4}}$, and

$$
\Re_{0}^{H}=\frac{\alpha^{*} \epsilon_{1} \omega_{1} M_{2}\left(\left(1-\beta^{*}\right) \epsilon_{1}^{*} \eta\left(k_{23} k_{24}+l_{2}\left(k_{24}+\eta_{15}\right)\right)+\left(1-p_{2}\right) \epsilon_{1}^{*} \beta^{*} \eta k_{22}\left(k_{24}+\eta_{15}\right)+k_{22} k_{23} \epsilon_{1}^{*} \beta^{*} \eta p_{2}\right)}{N_{H}\left(\alpha_{4}+\mu+\mu_{1}\right) k_{21} k_{22} k_{23} k_{24}},
$$

where $k_{21}=\alpha_{4}+\epsilon_{1}^{*} \eta+\mu+\mu_{1}, k_{22}=l_{2}+\mu+\mu_{1}+d_{2}+\eta_{12}+t_{3} \alpha_{4}, k_{23}=\mu+\mu_{1}+t_{2}^{\prime} d_{2}+\eta_{15}+m_{2}+t_{3} \alpha_{4}$, and $k_{24}=\mu+\mu_{1}+t_{2}^{*} d_{2}+\eta_{12}^{*}+t_{3} \alpha_{4}$.

Applying an analogous procedure to the TB-Only sub-model we obtain the following result:

Lemma 9. The infection-free equilibrium point, $\epsilon_{0}^{H}$ is asymptotically stable if $\Re_{0}^{H}<1$ and unstable if $\Re_{0}^{H}>1$. 


\section{TB-Diabetes sub-model}

The sub-model that relates diabetes to TB is obtained when $S_{H}=S_{T}=E_{H}=E_{T}=I_{H_{1}}=I_{H_{2}}=I_{T_{1}}=I_{T_{2}}=$ $R_{H_{1}}=R_{H}=R_{T_{1}}=R_{T}=0$ and is given by the system:

$$
\begin{aligned}
& { }^{c} \mathbb{D}_{t}^{\alpha} S_{D}=M_{3}-\left(\alpha_{2}+\mu+\mu_{2}+\omega_{2} \lambda_{T}\right) S_{D} \\
& { }^{c} \mathbb{D}_{t}^{\alpha} E_{D}=\beta_{1}^{\prime} \omega_{2} \lambda_{D} R_{D}+\omega_{2} \lambda_{T} S_{D}-\left(\eta+\mu+\mu_{2}+\alpha_{2}\right) E_{D}, \\
& { }^{c} \mathbb{D}_{t}^{\alpha} I_{D_{1}}=\left(1-\beta^{*}\right) \epsilon_{2}^{*} \eta E_{D}-\left(l_{3}+t_{2} \alpha_{2}+\mu+\mu_{2}+d_{3}+\eta_{13}\right) I_{D_{1}}, \\
& { }^{c} \mathbb{D}_{t}^{\alpha} I_{D_{2}}=\left(1-p_{3}\right) \epsilon_{2}^{*} \beta^{*} \eta E_{D}+l_{3} \eta I_{D_{1}}-\left(t_{2} \alpha_{2}+m_{3}+\mu+\mu_{2}+t_{3}^{\prime} d_{3}+\eta_{16}\right) I_{D_{2}}, \\
& { }^{c} \mathbb{D}_{t}^{\alpha} R_{D_{1}}=p_{3} \beta^{*} \epsilon_{2}^{*} \eta E_{D}+\eta_{16} I_{D_{2}}-\left(\eta_{13}^{*}+t_{2} \alpha_{2}+\mu+\mu_{2}+t_{1}^{*} d_{1}\right) R_{D_{1}}, \\
& { }^{c} \mathbb{D}_{t}^{\alpha} R_{D}=m_{3} I_{D_{2}}+\eta_{13} I_{D_{1}}+\eta_{13}^{*} R_{D_{1}}-\left(\alpha_{2}+\mu+\mu_{2}+\beta_{1}^{\prime} \omega_{2} \lambda_{D}\right) R_{D}
\end{aligned}
$$

with non-negative initial conditions and

$$
\lambda_{D}=\alpha^{*} \frac{\epsilon_{2}\left(I_{D_{1}}+I_{D_{2}}+R_{D_{1}}\right)}{N_{D}}
$$

where $N_{D}=S_{D}+E_{D}+I_{D_{1}}+I_{D_{2}}+R_{D_{1}}+R_{D}$.

Considering biological constraints, the system (15) will be studied in the following region biologically feasible:

$$
D_{3}=\left\{\left(S_{D}, E_{D}, I_{D_{1}}, I_{D_{2}}, R_{D_{1}}, R_{D}\right) \in \mathbb{R}_{+}^{6}: N_{D}(t) \leq \frac{M_{3}}{\mu}\right\} .
$$

It can be easily shown that solution $\left(S_{D}(t), E_{D}(t), I_{D_{1}}(t), I_{D_{2}}(t), R_{D_{1}}(t), R_{D}(t)\right)$ of the system are bounded and positively invariant.

The disease-free equilibrium point is $\epsilon_{0}^{D}=\left(S_{0}^{D}, 0,0,0,0,0\right), \quad$ where $S_{0}^{D}=\frac{M_{3}}{\mu+\mu_{2}+\alpha_{2}}$, and

$$
\Re_{0}^{D}=\frac{\alpha^{*} \epsilon_{2} \omega_{2} M_{3}\left(\left(1-\beta^{*}\right) \epsilon_{2}^{*} \eta\left(k_{33} k_{34}+l_{3}\left(k_{34}+\eta_{16}\right)\right)+\left(1-p_{3}\right) \epsilon_{2}^{*} \beta^{*} \eta k_{32}\left(k_{34}+\eta_{16}\right)+k_{32} k_{33} \epsilon_{2}^{*} \beta^{*} \eta p_{3}\right)}{N_{D}\left(\alpha_{2}+\mu+\mu_{2}\right) k_{31} k_{32} k_{33} k_{34}},
$$

where $k_{31}=\alpha_{2}+\epsilon_{2}^{*} \eta+\mu+\mu_{2}, k_{32}=l_{3}+\mu+d_{3}+\eta_{13}+t_{2} \alpha_{2}+\mu_{2}, k_{33}=\mu+t_{3}^{\prime} d_{3}+\eta_{16}+m_{3}+t_{2} \alpha_{2}+\mu_{2}$, and $k_{34}=\mu+\mu_{2}+t_{3}^{*} d_{3}+\eta_{13}^{*}+t_{2} \alpha_{2}$.

The following result is proved by applying the analogous methodology to the sub-model TB-Only.

Lemma 10. The infection-free equilibrium point, $\epsilon_{0}^{D}$ is asymptotically stable if $\Re_{0}^{D}<1$ unstable if $\Re_{0}^{D}>1$.

\subsection{Analysis of the full model}

For the full model (1), infection-free equilibrium point is

$$
\epsilon_{0}^{G}=\left(S_{0}^{T}, S_{0}^{H}, S_{0}^{D}, 0,0,0,0,0,0,0,0,0,0,0,0,0,0,0\right)
$$

and we calculate the basic reproduction number as in the previous sub-models by the next-generation operator method. The dominant eigenvalues of the next-generation matrix are $\Re_{0}^{T}, \Re_{0}^{H}$ and $\Re_{0}^{D}$. Then, the basic reproduction number of the model (1) is

$$
\Re_{0}=\max \left\{\Re_{0}^{T}, \Re_{0}^{H}, \Re_{0}^{D}\right\} .
$$

\section{Global Stability}

Now, we list two conditions that if, also guarantee the global asymptotic stability of the disease-free equilibrium point. Following [31], we can rewrite the model (1) as

$$
\begin{aligned}
& { }^{c} \mathbb{D}_{t}^{\alpha} S=F(S, I), \\
& { }^{c} \mathbb{D}_{t}^{\alpha} I=G(S, I), \quad G(S, 0)=0,
\end{aligned}
$$


where $S \in \mathbb{R}_{+}^{6}$ is the vector whose components are the number of uninfected and recovered and $I \in \mathbb{R}_{+}^{12}$ denotes the number of infected individuals including the latent and the infectious (the other variables of the model (1)).

The disease-free equilibrium is now denoted by $E_{0}^{G}=\left(S_{0}^{*}, 0\right), S_{0}^{*}=\left(S_{0}, 0,0,0\right), S_{0}=\left(S_{0}^{T}, S_{0}^{H}, S_{0}^{D}\right)$ where $S_{0}^{T}=$ $\frac{M_{1}}{\mu+\alpha_{1}+\alpha_{2}}, S_{0}^{H}=\frac{M_{2}}{\mu+\mu_{1}+\alpha_{4}}$ and $S_{0}^{D}=\frac{M_{3}}{\mu+\mu_{2}+\alpha_{2}}$.

The conditions $H_{1}$ and $H_{2}$ below must be satisfied to guarantee the global asymptotic stability of $E_{0}^{G}$.

$$
\begin{array}{ll}
H_{1}: & \text { For }{ }^{c} \mathbb{D}_{t}^{\alpha} S=F(S, 0), \quad S_{0}^{*} \quad \text { is globally asymptotically stable, } \\
H_{2}: & G(S, I)=A I-G^{*}(S, I), \quad G^{*}(S, I) \geq 0, \quad \text { for } \quad(S, I) \in \Omega,
\end{array}
$$

where $A=D_{I} G\left(S_{0}^{*}, 0\right)\left(D_{I} G\left(S_{0}^{*}, 0\right)\right.$ is the Jacobian of $\mathrm{G}$ at $\left.\left(S_{0}^{*}, 0\right)\right)$ is a M-matrix (the off-diagonal elements of $A$ are non-negative) and $\Omega$ is the biologically feasible region.

For global stability, we have:

Theorem 11. The fixed point $E_{0}^{G}$ is a globally asymptotically stable equilibrium of model (1) provided that $\Re_{0}<1$ and that the conditions $\mathrm{H}_{1}$ and $\mathrm{H}_{2}$ are satisfied.

This theorem and an analogous proof can be found in the bibliographical reference [32].

proof. Let

$$
F(S, 0)=\left(\begin{array}{c}
M_{1}-\left(\mu+\alpha_{1}+\alpha_{2}\right) S_{T} \\
M_{2}-\left(\mu+\mu_{1}+\alpha_{4}\right) S_{H} \\
M_{3}-\left(\mu+\mu_{2}+\alpha_{2}\right) S_{D} \\
0 \\
0 \\
0
\end{array}\right) .
$$

As $F(S, 0)$ is a linear equation, we have that $S_{0}^{*}$ is globally stable, hence $H_{1}$ is satisfied. Then,

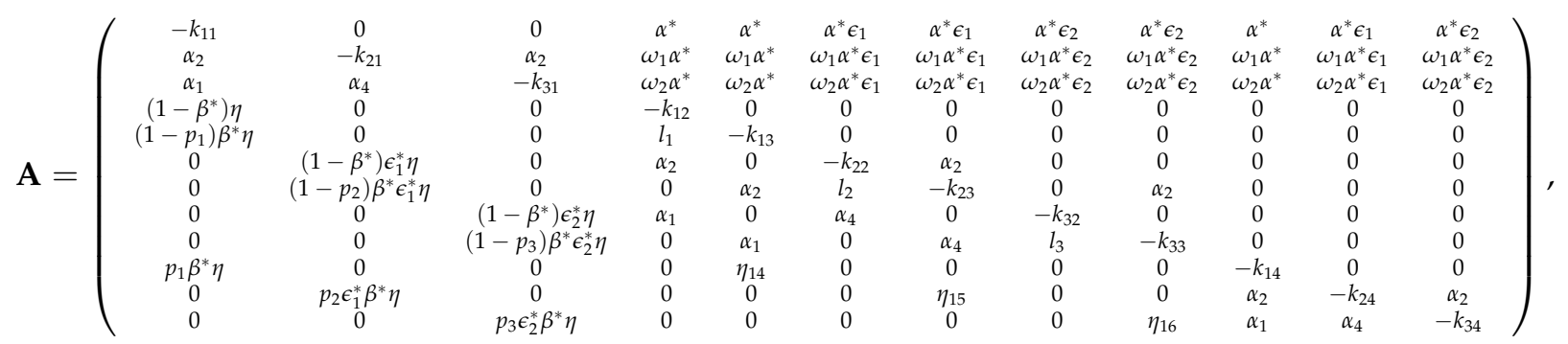

$$
\mathbf{I}=\left(E_{T}, E_{H}, E_{D}, I_{T_{1}}, I_{T_{2}}, I_{H_{1}}, I_{H_{2}}, I_{D_{1}}, I_{D_{2}}, R_{T_{1}}, R_{H_{1}}, R_{D_{1}}\right),
$$

$G^{*}(S, I)=A I^{T}-G(S, I)$,

$G^{*}(S, I)=\left(\begin{array}{l}G_{1}^{*}(S, I) \\ G_{2}^{*}(S, I) \\ G_{3}^{*}(S, I) \\ G_{4}^{*}(S, I) \\ G_{5}^{*}(S, I) \\ G_{6}^{*}(S, I) \\ G_{7}^{*}(S, I) \\ G_{8}^{*}(S, I) \\ G_{9}^{*}(S, I) \\ G_{10}^{*}(S, I) \\ G_{11}^{*}(S, I) \\ G_{12}^{*}(S, I)\end{array}\right)$ 


$$
=\left(\begin{array}{c}
\alpha^{*}\left(I_{T_{1}}+I_{T_{2}}+R_{T_{1}}+\epsilon_{1}\left(I_{H_{1}}+I_{H_{2}}+R_{H_{1}}\right)+\epsilon_{2}\left(I_{D_{1}}+I_{D_{2}}+R_{D_{1}}\right)\right)\left(1-\frac{S_{T}+\beta_{1}^{\prime} R_{T}}{N}\right) \\
\omega_{1} \alpha^{*}\left(I_{T_{1}}+I_{T_{2}}+R_{T_{1}}+\epsilon_{1}\left(I_{H_{1}}+I_{H_{2}}+R_{H_{1}}\right)+\epsilon_{2}\left(I_{D_{1}}+I_{D_{2}}+R_{D_{1}}\right)\right)\left(1-\frac{S_{H}+\beta_{1}^{\prime} R_{H}}{N}\right) \\
\omega_{2} \alpha^{*}\left(I_{T_{1}}+I_{T_{2}}+R_{T_{1}}+\epsilon_{1}\left(I_{H_{1}}+I_{H_{2}}+R_{H_{1}}\right)+\epsilon_{2}\left(I_{D_{1}}+I_{D_{2}}+R_{D_{1}}\right)\right)\left(1-\frac{S_{D}+\beta_{1}^{\prime} R_{D}}{N}\right) \\
0 \\
0 \\
0 \\
0 \\
0 \\
0 \\
0 \\
0 \\
0
\end{array}\right) .
$$

Since $S_{T}+\beta_{1}^{\prime} R_{T}, S_{H}+\beta_{1}^{\prime} R_{H}$ and $S_{D}+\beta_{1}^{\prime} R_{D}$ are always less than or equal to $N, \frac{S_{T}+\beta_{1}^{\prime} R_{T}}{N} \leq 1, \frac{S_{H}+\beta_{1}^{\prime} R_{H}}{N} \leq 1$ and $\frac{S_{D}+\beta_{1}^{\prime} R_{D}}{N} \leq 1$. Thus $G^{*}(S, I) \geq 0$ for all $(S, I) \in D$. The $E_{0}^{G}$ is a globally asymptotically stable.

\section{Numerical results}

The goal of this section is to simulate the model (1). The numerical results of the Caputo derivative are obtained by the predictor-corrector PECE method of Adams-Bashforth-Moulton type [33, 34]. We use 10 years as the time horizon. The simulations are conducted with different values of the order of the fractional derivative $\alpha=$ $0.3,0.5,0.7,0.9,1$. The Table 2 shows the values used as the initial conditions and parameters for the simulations. We present the results of the simulations for the resistance compartments.

Table 2. Values of variables and parameters used in the model (1)

\begin{tabular}{|c|c|c|c|c|c|}
\hline Variables & Value & Variables & Valuee & Variables & Value \\
\hline$S_{T}(0)$ & 8741400 & $S_{H}(0)$ & 111000 & $S_{D}(0)$ & 200000 \\
\hline$E_{T}(0)$ & 565600 & $E_{H}(0)$ & 5000 & $E_{D}(0)$ & 8500 \\
\hline$I_{T_{1}}(0)$ & 20000 & $I_{H_{1}}(0)$ & 1400 & $I_{D_{1}}(0)$ & 1800 \\
\hline$I_{T_{2}}(0)$ & 1300 & $I_{H_{2}}(0)$ & 400 & $I_{D_{2}}(0)$ & 550 \\
\hline$R_{T_{1}}(0)$ & 700 & $R_{H_{1}}(0)$ & 210 & $R_{D_{1}}(0)$ & 250 \\
\hline$R_{T}(0)$ & 8800 & $R_{H}(0)$ & 500 & $R_{D}(0)$ & 300 \\
\hline Parameters & Value & Reference & Parameters & Value & Reference \\
\hline$M_{1}, M_{2}, M_{3}$ & $667685,10000,50000$ & [35], Assumed, Assumed & $\alpha^{*}$ & 9.5 & {$[35-37]$} \\
\hline$\alpha_{1}, \alpha_{2}$ & $0.0075,0.009$ & Assumed, $[35,36]$ & $\omega_{1}, \omega_{2}$ & $1.22,1.10$ & {$[35,36,38]$, assumed } \\
\hline$\alpha_{4}$ & 17.3 (per thousand people per year) & [39] & $\epsilon_{1}, \epsilon_{2}$ & $1.3,1.1$ & Assumed, $[35,36]$ \\
\hline$\mu, \mu_{1}, \mu_{2}$ & $\begin{array}{l}1 / 53.5,0.045,0.03 \\
0.275,0.33,1.5 * d_{1}\end{array}$ & {$[35,36,38]$, Assumed } & $\eta, \beta^{*}$ & $\begin{array}{l}0.5,0.04 \\
1.3,1.1\end{array}$ & $\begin{array}{l}{[35,36,38,40,41]} \\
{[381, \text { Assumed }}\end{array}$ \\
\hline$t_{1}^{*}, t_{2}^{*}, t_{3}^{*}$ & $1.01,1.02,1.01$ & Assumed & $t_{1}^{\prime}, t_{2}^{\prime}, t_{3}^{\prime}$ & $1,1.01,1$ & Assumed \\
\hline$\beta_{1}^{\prime}$ & 0.9 & [38] & $l_{1}, l_{2}, l_{3}$ & $0.0018,0.0022,0.0048$ & {$[37,42-44]$, Assumed } \\
\hline$m_{1}, m_{2}, m_{3}$ & $0.6266,0.45,0.4054$ & {$[35,36]$, Assumed } & $\eta_{14}, \eta_{15}, \eta_{16}$ & $0.013,0.022,0.006$ & {$[37,42-44]$, Assumed } \\
\hline$\eta_{11}, \eta_{12}, \eta_{13}$ & $0.7372,0.55,0.7372$ & {$[35,36]$, Assumed } & $p_{1}, p_{2}, p_{3}$ & $0.00225,0.0035,0.0041$ & {$[40,41,45]$, Assumed } \\
\hline$\eta_{11}^{*}, \eta_{12}^{*}, \eta_{13}^{*}$ & $0.4006,0.255,0.3317$ & {$[35,36]$, Assumed } & $t_{1}, t_{2}, t_{3}$ & $1.01,1.01,1.01$ & Assumed \\
\hline
\end{tabular}

For MDR-TB cases in all subpopulations at the beginning of the study, we have a decrease in the number of cases reported. Initially, fewer cases are reported for the lower $\alpha$-values (lower $\alpha$-values implies fewer cases). At about the year of study (depending on the sub-population) behavior changes and higher $\alpha$-values report lower numbers of cases, see Figures $1 \mathrm{~b}, 1 \mathrm{~d}$ and $1 \mathrm{f}$. Then a growth begins in all sub-populations of MDR-TB and at the end of the study period, a higher number of cases was reported for the highest $\alpha$-values, see Figures 1a, 1c and 1e. The highest number of MDR-TB cases was reported by the TB-Only sub-population followed by the TB-Diabetes sub-population throughout the study period and for the different $\alpha$-values. Due to these results, it is recommended to apply MDR-TB control in all sub-populations at the beginning of the study period to control the growth in the number of cases.

The XDR-TB cases in the TB-Only sub-population decreased throughout the study period. At the beginning of the study, fewer cases were reported in less time for lower $\alpha$-values but at the end of the study period, the opposite occurred (higher $\alpha$-values reported a lower number of cases). We must pay attention when $\alpha=1$ because at the end of the study there is a slight increase in the number of cases, see Figures $2 a$ and $2 b$. 


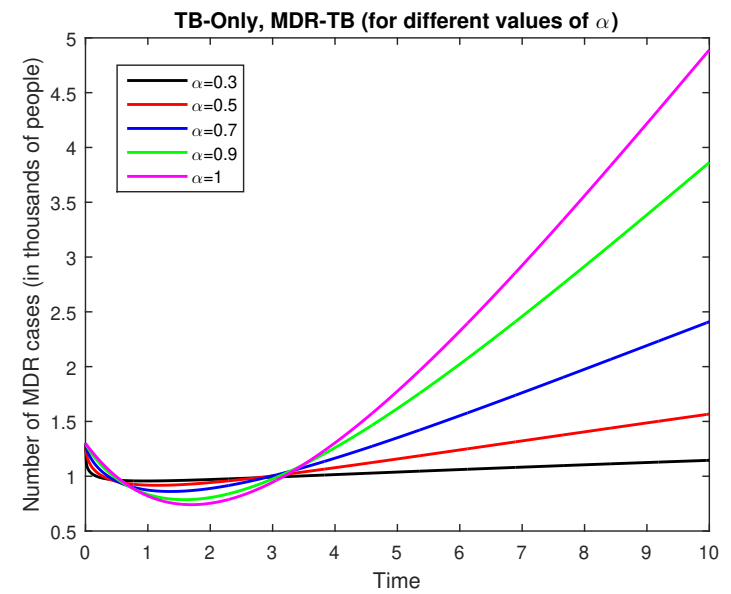

(a) MDR-TB cases in the TB-Only, study period 10 years

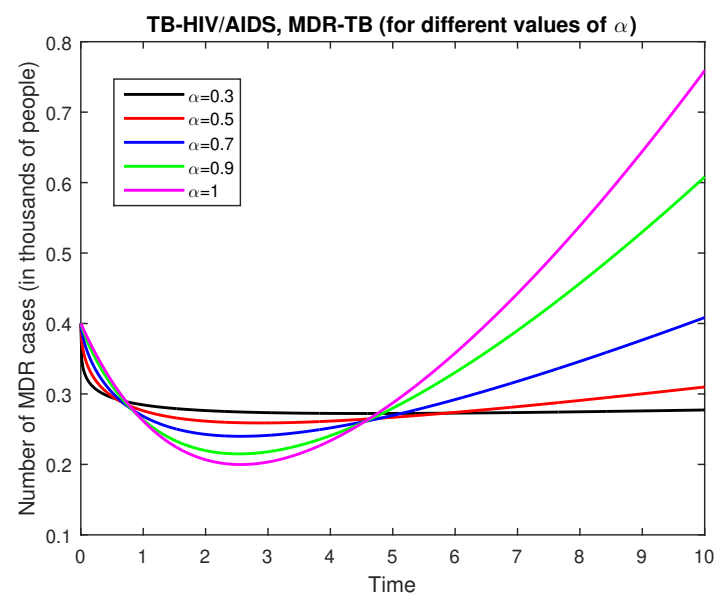

(c) MDR-TB cases in the TB-HIV/AIDS subpopulation, study period 10 years

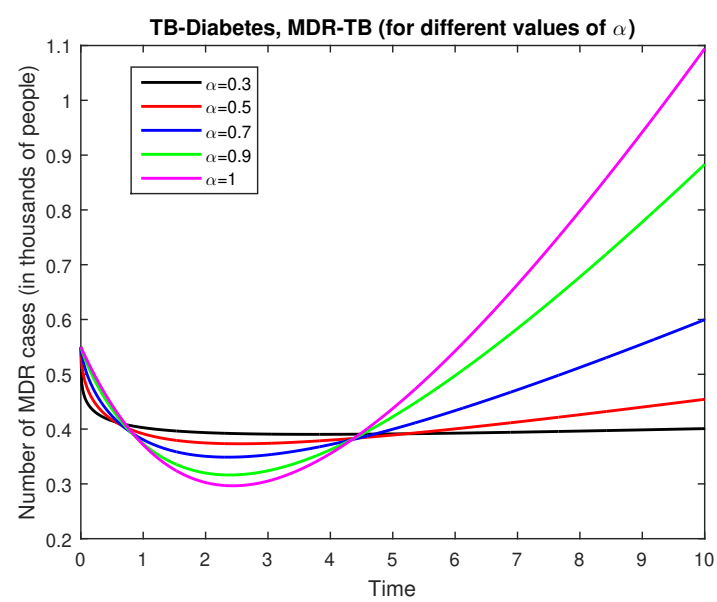

(e) MDR-TB cases in the TB-Diabetes subpopulation, study period 10 years

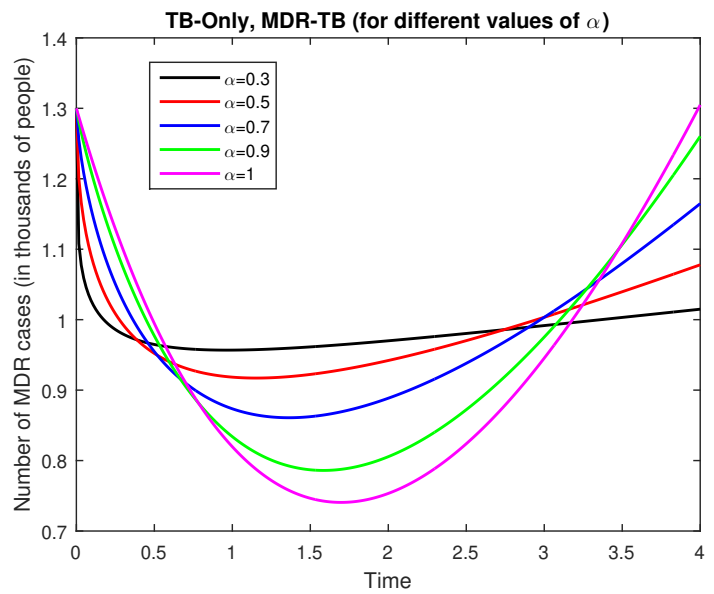

(b) MDR-TB cases in the TB-Only, study period 4 years

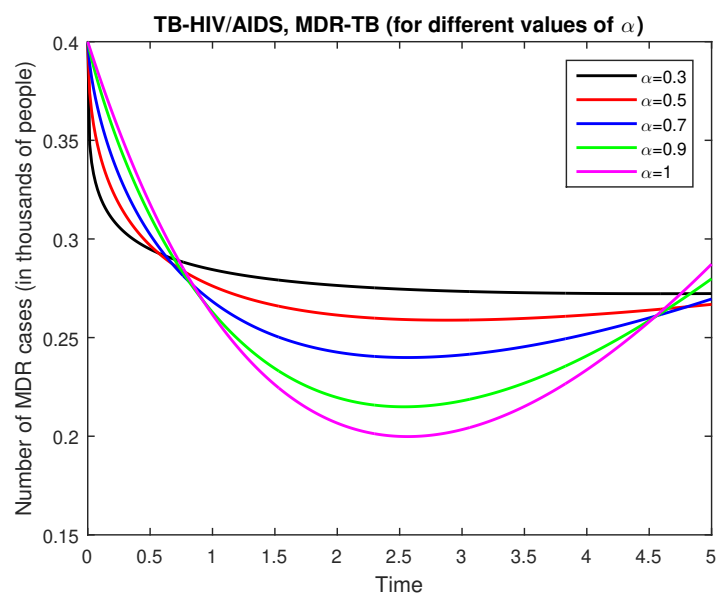

(d) MDR-TB cases in the TB-HIV/AIDS subpopulation, study period 5 years

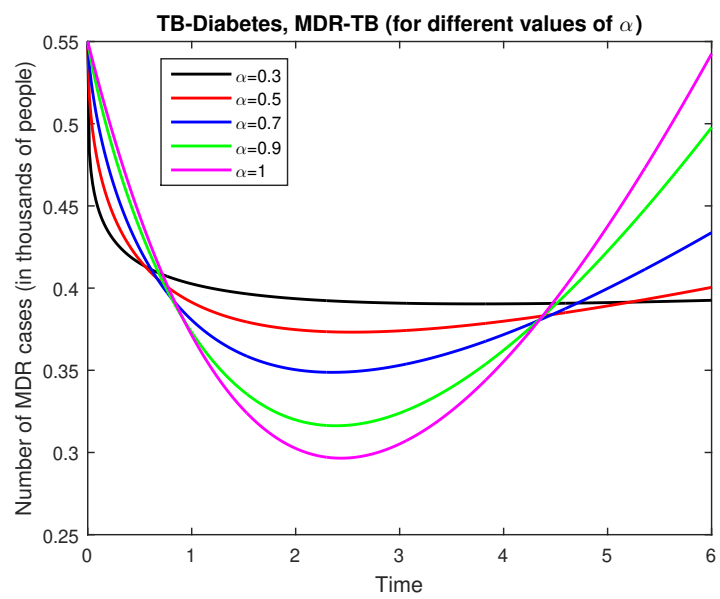

(f) MDR-TB cases in the TB-Diabetes subpopulation, study period 6 years

Figure 1. Behavior of MDR-TB cases for different $\alpha$-values over time 


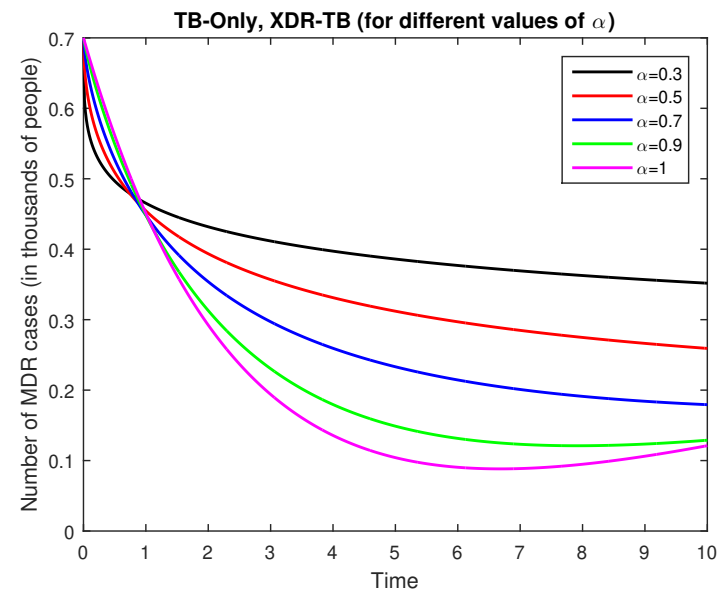

(a) XDR-TB cases in the TB-Only, study period 10 years

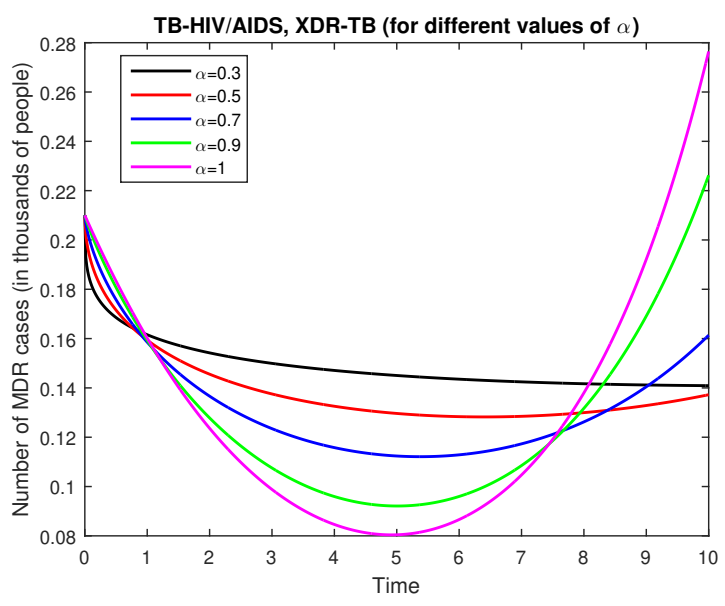

(c) XDR-TB cases in the TB-HIV/AIDS subpopulation, study period 10 years

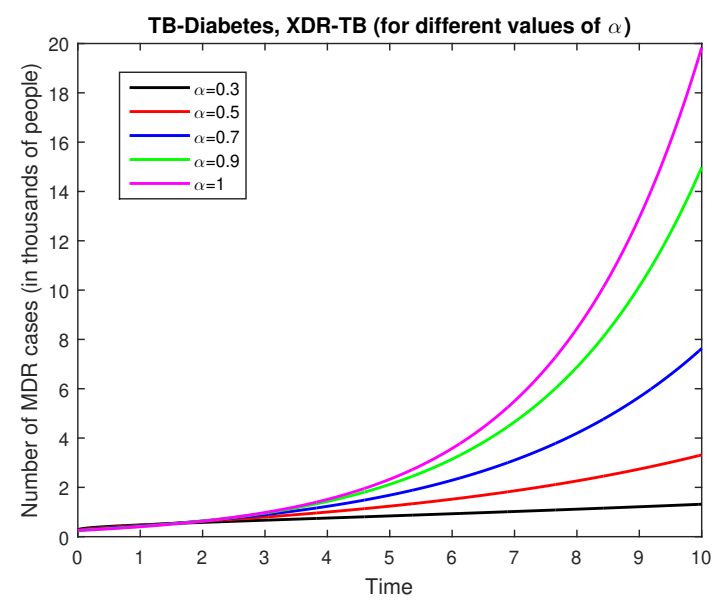

(e) XDR-TB cases in the TB-Diabetes subpopulation, study period 10 years

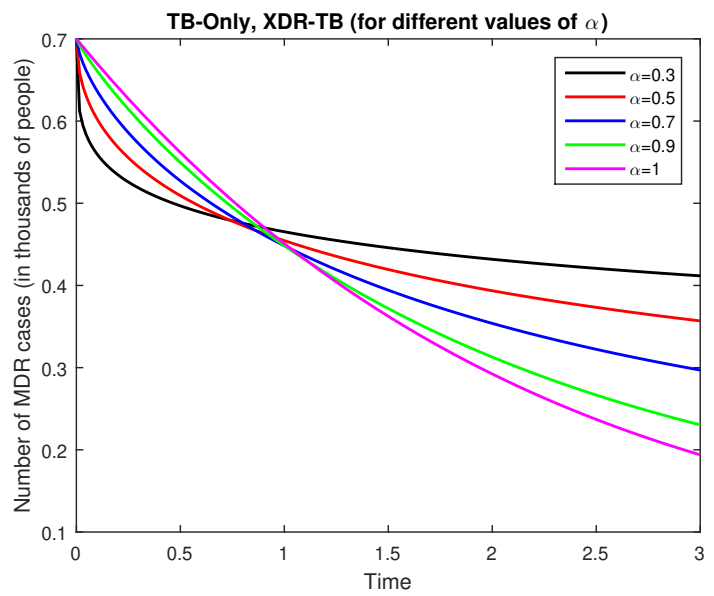

(b) XDR-TB cases in the TB-Only, study period 3 years

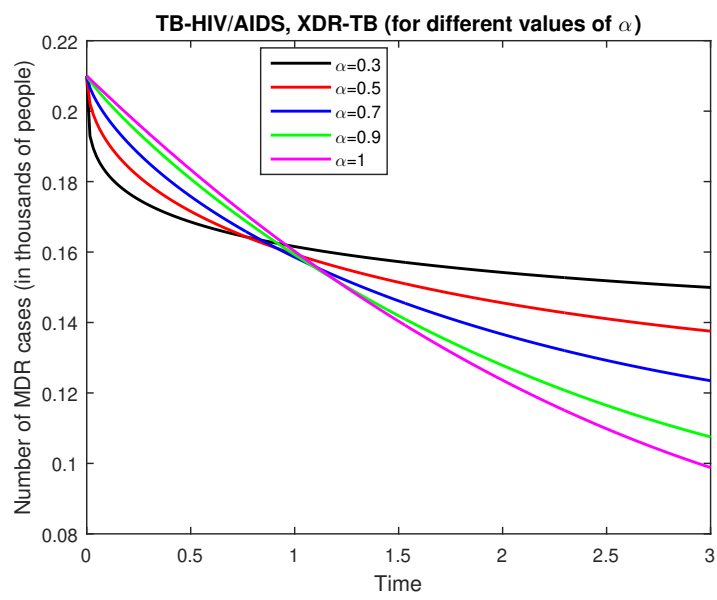

(d) XDR-TB cases in the TB-HIV/AIDS subpopulation, study period 3 years

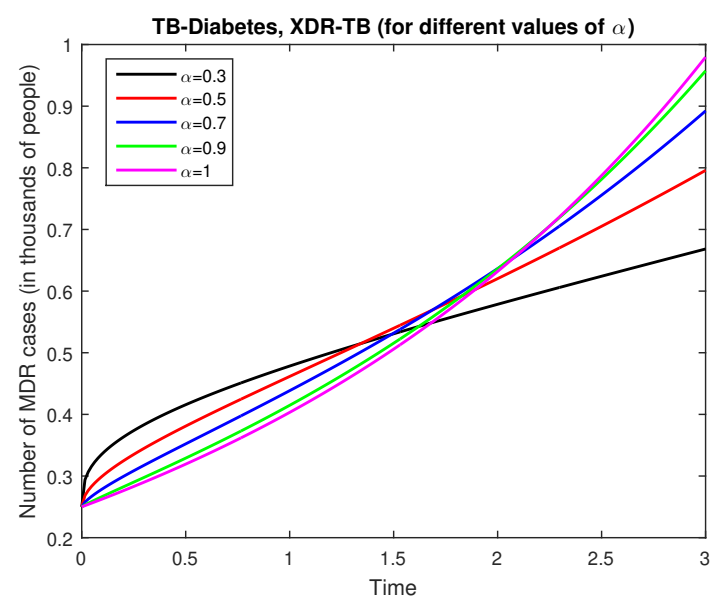

(f) XDR-TB cases in the TB-Diabetes subpopulation, study period 3 years

Figure 2. Behavior of XDR-TB cases for different $\alpha$-values over time 
In the TB-HIV/AIDS sub-population at the beginning of the study, there is a decrease in the number of cases where lower $\alpha$-values report fewer cases (up to approximately one year of study). Over time, the decrease in the number of cases continues, but now with higher $\alpha$-values the number of cases is lower. Approximately 5 years into the study, we have an increase in the number of cases for $\alpha>0.5$ and at the end of the period of study, we have differentiated results for the different $\alpha$. For $\alpha>0.5$, higher $\alpha$-values the number of reported cases is higher. For $\alpha>0.5$, higher $\alpha$-values reported a higher number of cases. For $\alpha \leq 0.5$, the opposite is true, at lower $\alpha$ values the number of reported cases is higher, see Figures $2 \mathrm{c}$ and $2 \mathrm{~d}$. This event is important to take into account for the application of an effective control strategy in this sub-population. We recommend applying control from the beginning of the study in order to avoid the growth of cases taking advantage of the initial decrease in these compartments.

In the TB-Diabetes sub-population, there was an increase in the number of cases during the full study period. At the beginning of the study, the highest number of cases was reported for lower $\alpha$-values, see Figure $2 \mathrm{f}$. At the end of the study period, it happens that higher $\alpha$-values report a higher number of cases, see Figure 2e. In general, the highest number of XDR-TB cases was reported from the TB-Diabetes sub-population. We recommend applying an effective control strategy in this sub-population with the objective of reducing the number of XDR-TB cases due to the growth of cases throughout the study period for all $\alpha$-values.

During the computational experimentation, we found that the MDR-TB compartments have a decrease at the beginning of the study period and a growth at the end for the different $\alpha$-values, which allows us to design a control strategy with the objective of avoiding the growth of the number of cases. For XDR-TB cases, we recommend paying attention to the TB-HIV/AIDS and TB-Diabetes sub-populations due to the growing number of cases. In particular to the diabetic XDR-TB cases because they report the highest number of cases among all TB treatment resistance compartments.

\section{Conclusions}

In this paper, a mathematical model for the efficacy of TB treatment taking into account its relationship with HIV / AIDS and diabetes that is in [25] using fractional order derivatives in the Caputo sense is formulated. We studied MDR-TB and XDR-TB in the different sub-populations TB-Only, TB-HIV/AIDS, and TB-Diabetes. We calculated and analyzed the break-even points of the different sub-populations using the next-generation matrix method. The study of the basic reproduction number per sub-population allows us to know how the epidemic will behave specifically in these sub-populations and to obtain information on the full model (1), since the basic number of reproduction of the full model is defined as the maximum of the basic reproduction number of the sub-populations $\left(\Re_{0}=\max \left\{\Re_{0}^{T}, \Re_{0}^{H}, \Re_{0}^{D}\right\}\right)$. If $\Re_{0}=\max \left\{\Re_{0}^{T}, \Re_{0}^{H}, \Re_{0}^{D}\right\}>1$, this implies that at least one (can be all) of the basic reproduction numbers will be greater than unity, thus the infection will be able to start spreading in a population and specifically in sub-populations with basic reproduction number greater than unity. If $\Re_{0}=\max \left\{\Re_{0}^{T}, \Re_{0}^{H}, \Re_{0}^{D}\right\}<1$ this implies that the basic reproduction number by subpopulations and overall are less than unity with we have that the infection will die out in the long run. The results 8-11 show the behavior of the infection-free equilibrium points for the sub-populations and the general population depending on the value of the basic reproduction number.

For the computational simulations, we used a predictor-corrector method in order to study the behavior of the different resistance compartments over time for different $\alpha$-values. From the results of the simulations, we have that in MDR-TB at the beginning of the study, there is a decrease of cases in all sub-populations. Then the number of cases begins to grow and at the end of the study period in MDR-TB, a higher number of cases is obtained for the higher $\alpha$-values. For MDR-TB control, we recommended controlling from the beginning of the study to avoid the growth in the number of cases. Throughout the study, the number of XDR-TB cases in the TB-Only sub-population decreased. The XDR-TB cases in the TB-HIV sub-population initially have a decrease in the number of cases. Approximately 5 years into the study there is a growth in the number of cases for $\alpha>0.5$ and at the end of the study, there is a differentiated behavior, for $\alpha>0.5$ the higher $\alpha$-values reported a higher number of cases and for $\alpha \leq 0.5$ the lower the $\alpha$-values the higher number of cases reported. This factor must be taken into account to design an effective control strategy. In the TB-Diabetes sub-population throughout the study period, there is an increase in the number of XDR-TB cases. At the beginning for lower $\alpha$-values the number of cases is higher and at the end of the period, the opposite situation occurs. The diabetic XDR-TB are the highest number of resistance cases compared to all TB treatment resistance compartments. We recommend paying special attention to the control in this compartment due to its growth. This work allowed us to study the model for derivatives of different orders, taking advantage of the facilities that fractional derivatives allow us. Computer simulations provide information for an effective design of a control strategy for Tuberculosis 
(treatment resistance and transmission). In future work, we will study the optimal control problem of reducing resistance to TB treatment with this model, and perform computational simulations in real scenarios.

\section{References}

[1] G. Delogu, M. Sali, and G. Fadda1, “The Biology of Mycobacterium Tuberculosis Infection," Mediterranean Journal of Hematology and Infectious Diseases, vol. 5, no. 1, p. e2013070, 2013.

[2] A. K. Niazi and S. Kalra, "Diabetes and tuberculosis: a review of the role of optimal glycemic control," Journal of Diabetes $\mathcal{E}$ Metabolic Disorders, vol. 11, p. 28, 2012.

[3] C. R. Stevenson, J. A. Critchley, N. G. Forouhi, G. Roglic, B. G. Williams, C. Dye, and N. C. Unwin, “Diabetes and the risk of tuberculosis: a neglected threat to public health?" Chronic Illness, vol. 3, no. 3, pp. 228-45, 2007.

[4] P. Baghaei, M. Marjani, P. Javanmard, P. Tabarsi, and M. R. Masjedi, "Diabetes mellitus and tuberculosis facts and controversies," Journal of Diabetes E Metabolic Disorders, vol. 12, no. 1, p. 58, 2013.

[5] R. M. Guimarães, A. P. Lobo, E. A. Siqueira, T. F. Borges, and S. C. Melo, "Tuberculosis, HIV, and poverty: temporal trends in Brazil, the Americas, and worldwide," Jornal Brasileiro de Pneumologia, vol. 38, pp. 511-517, 2012.

[6] L. C. de Barros, M. M. Lopes, F. S. Pedro, E. Esmi, J. P. C. dos Santos, and D. E. Sánchez, "The memory effect on fractional calculus: an application in the spread of COVID-19," Computational and Applied Mathematics, vol. 40, no. 3, p. 72, 2021.

[7] Fatmawati, M. A. Khan, E. Bonyah, Z. Hammouch, and E. M. Shaiful, "A mathematical model of tuberculosis (TB) transmission with children and adults groups: A fractional model," AIMS Mathematics, vol. 5, no. 4, pp. 2813-2842, 2020.

[8] M. Saeedian, M. Saeedian, M. Khalighi, Azimi-Tafreshi, and M. Ausloos, "Memory effects on epidemic evolution: The susceptible-infected-recovered epidemic model," Physical Review E, vol. 95, no. 2, p. 022409, 2017.

[9] Fatmawati, M. A. Khan, E. Bonyah, Z. Hammouch, and E. M. Shaiful, "The fractional-order SIR and SIRS epidemic models with variable population size," Journal of the Egyptian Mathematical Society, vol. 22, no. 1, pp. 50-54, 2014.

[10] B. B. Beatrix, D. Haydon, and K. Cuddington, Hysteresis, in book: Encyclopedia of Ecology. Elsevier Science, 2008.

[11] T. K. Yamana, X. Qiu, and E. A. B. Eltahir, "Hysteresis in simulations of malaria transmission," Advances in Water Resources, vol. 108, pp. 416-422, 2017.

[12] A. Pimenov, T. Kelly, A. Korobeinikov, M. O'Callaghan, A. Pokrovskii, and D. Rachinskii, “Memory effects in population dynamics: Spread of infectious disease as a case study," Mathematical Modelling of Natural Phenomena, vol. 7, no. 3, pp. 204-226, 2012.

[13] I. Ullah, S. Ahmad, M. ur Rahman, and M. Arfan, "Investigation of fractional order tuberculosis (TB) model via Caputo derivative," Chaos, Solitons E Fractals, vol. 142, p. 110479, 2011.

[14] S. Rosa and D. F. M. Torres, "Optimal Control and Sensitivity Analysis of a Fractional Order TB Model," Statistics, Optimization \& Information Computing, vol. 7, no. 3, pp. 617-625, 2019.

[15] M. Farman, M. Usman, A. Ahmad, and M. Ahmad, “Mathematical Analysis of Fractional Order Co-Infection TB and HIV Model," International Journal of Analysis and Applications, vol. 18, no. 1, pp. 16-32, 2020.

[16] H. Khan, J. Gómez-Aguilar, A. Alkhazzan, and A. Khan, "A fractional order HIV-TB coinfection model with nonsingular Mittag-Leffler Law," International Journal of Analysis and Applications, vol. 43, no. 6, pp. 3786-3806, 2020.

[17] C. M. A. Pinto and A. R. M. Carvalho, "A latency fractional order model for HIV dynamics," Journal of Computational and Applied Mathematics, vol. 312, pp. 240-256, 2020.

[18] Fatmawati, E. M. Shaiful, and M. I. Utoyo, "A Fractional-Order Model for HIV Dynamics in a Two-Sex Population," International Journal of Mathematics and Mathematical Sciences, vol. 2018, p. Article ID 6801475, 2018.

[19] A. R. M. Carvalho, C. M. A. Pinto, and D. Baleanu, "HIV/HCV coinfection model: a fractional-order perspective for the effect of the HIV viral load," Advances in Difference Equations, vol. 2018, p. 2, 2018.

[20] M. U. Saleem, M. Farman, A. A. E. UlHaque, and M.O.Ahmad, "A Caputo Fabrizio fractional order model for control of glucose in insulin therapies for diabetes," Ain Shams Engineering Journal, vol. 11, no. 4, pp. 1309-1316, 2020.

[21] S. Sakulrang, E. J. Moore, S. Sungnul, and A. de Gaetano, "A fractional differential equation model for continuous glucose monitoring data," Advances in Difference Equations, vol. 2017, p. Article number: 150, 2017.

[22] I. Podlubny, The analysis of fractional differential equations, 1st ed. Academic Press, 1998, vol. 198.

[23] A. A. Kilbas, H. M. Srisvastava, and J. J. Trujillo, Theory and application fractional differential equation, 1st ed. Elsevier Science, 2006.

[24] R. Gorenflo, J. Loutschko, and Y. Luchko, "Computation of the Mittag-Leffler function $\mathbb{E}_{\alpha, \beta}(z)$ and its derivatives," Fractional Calculus and Applied Analysis, vol. 5, pp. 491-518, 2020.

[25] E. D. Moya, A. Pietrus, and S. M. oliva, "A mathematical model for the study of effectiveness in therapy in Tuberculosis taking into account associated diseases," Contemporary Mathematics, vol. 2, pp. 77-102, 2021.

[26] Z. Odibat and N. Shawagfeh, "A fractional calculus based model for the simulation of an outbreak of dengue fever," Applied Mathematics and Computation, vol. 186, no. 1, pp. 286-293, 2013.

[27] W. Lin, "Global existence theory and chaos control of fractional differential equations," Journal of Mathematical Analysis and Applications, vol. 332, no. 1, pp. 709-726, 2007.

[28] H. Kheiri and M. Jafari, "Stability analysis of a fractional order model for the hiv/aids epidemic in a patchy environment," Journal of Computational and Applied Mathematics, vol. 346, p. 323-339, 2019.

[29] P. van den Driessche and J. Watmough, "Reproduction numbers and sub-threshold endemic equilibria for compartmental models of disease transmission," Mathematical Biosciences, vol. 180, pp. 29-48, 2002. 
[30] M. S. Tavazoei and M. Haeri, "Chaotic attractors in incommensurate fractional order systems," Physica D: Nonlinear Phenomena, vol. 237, no. 20, pp. 2628-2637, 2008.

[31] C. Castillo-Chavez, Z. Feng, and W. Huang, "On the computation of R0 and its role on global stability," In: Mathematical Approaches for Emerging and Reemerging Infectious Diseases: An Introduction, Springer-Verlag, New York, pp. 229-250, 2002.

[32] C. M. A. Pinto and A. R. M. Carvalho, "Diabetes mellitus and TB co-existence: Clinical implications from a fractional order modelling," Applied Mathematical Modelling, vol. 68, pp. 219-243, 2019.

[33] K. Diethelm, N. J. Ford, and A. D. Freed, "A predictor-corrector approach for the numerical solution of fractional differential equations," Nonlinear Dynamics, vol. 29, pp. 3-22, 2002.

[34] K. Diethelm and A. D. Freed, The frac PECE subroutine for the numerical solution of differential equations of fractional order. Forschung und wissenschaftliches rechnen, 1998.

[35] A. R. Carvalho and C. M. Pinto, "Non-integer order analysis of the impact of diabetes and resistant strains in a model for TB infection," Communications in Nonlinear Science and Numerical Simulation, vol. 61, pp. 104-126, 2018.

[36] D. Moualeu, S. Bowong, J. Tewa, and Y. Emvudu, "Analysis of the impact of diabetes on The dynamical transmission of tuberculosis," Mathematical and Computer Modelling: An International Journal, vol. 7, no. 3, pp. 117 - 146, 2012.

[37] M. Zamir, G. Zaman, and A. S. Alshomrani, "Sensitivity analysis and optimal control of anthroponotic cutaneous leishmania," PLoS ONE, vol. 11, no. 8, p. e0160513, 2016.

[38] C. J. Silva and D. F. M. Torres, "A TB-HIV/AIDS coinfection model and optimal control treatment," SIAM Journal on Mathematical Analysis, vol. 35, no. 9, pp. 4639-4663, 2015.

[39] A. J. F. Cassetone, "Alterações metabólicas associadas ao uso de medicamento antirretroviral em pessoas vivendo com hiv/aids: caracterização e desenvolvimento de algoritmos inteligentes aplicados à sua identificação e previsão," Ph.D. dissertation, FMUSP, São Paulo, Brasil, 2017.

[40] C. Castillo-Chavez and Z. Feng, "To treat or not to treat: the case of tuberculosis," Journal of Mathematical Biology, vol. 35, no. 6, pp. 629-56, 1997.

[41] E. Jung, S. Lenhart, and Z. Feng, "Optimal control of treatments in a two-strain tuberculosis model," Journal of Mathematical Biology, vol. 2, no. 4, pp. 473-482, 2002.

[42] R. I. Hickson, G. N. Mercer, and K. M. Lokuge, "A metapopulation model of tuberculosis transmission with a case study from high to low burden areas," PloS ONE, vol. 7, no. 4, p. e34411, 2012.

[43] S. M. Blower, A. R. McLean, T. C. Porco, P. M. Small, P. C. Hopewell, M. A. Sanchez, and A. R. Moss, "The intrinsic transmission dynamics of tuberculosis epidemics," Nature Medicine, vol. 1, no. 8, pp. 815-21, 1995.

[44] T. Cohen, C. Colijn, B. Finklea, and M. Murray, "Exogenous re-infection and the dynamics of tuberculosis epidemics: local effects in a network model of transmission," Journal of The Royal Society Interface, vol. 4, no. 14, p. 523-531, 2007.

[45] M. Zamir, G. Zaman, and A. S. Alshomrani, "Sensitivity analysis and optimal control of anthroponotic cutaneous leishmania," PLoS ONE, vol. 11, no. 8, p. e0160513, 2016.

(C)2021 by the Author(s). This article is an open access article distributed under the terms and conditions of the Creative Commons Attribution-NonComercial 4.0 International License. Editorial of JJBM: Department of Mathematics, State University of Gorontalo, Jln. Prof. Dr. Ing. B. J. Habibie, Bone Bolango 96119, Indonesia. 


\section{Submit your manuscript at http://ejurnal.ung.ac.id/}

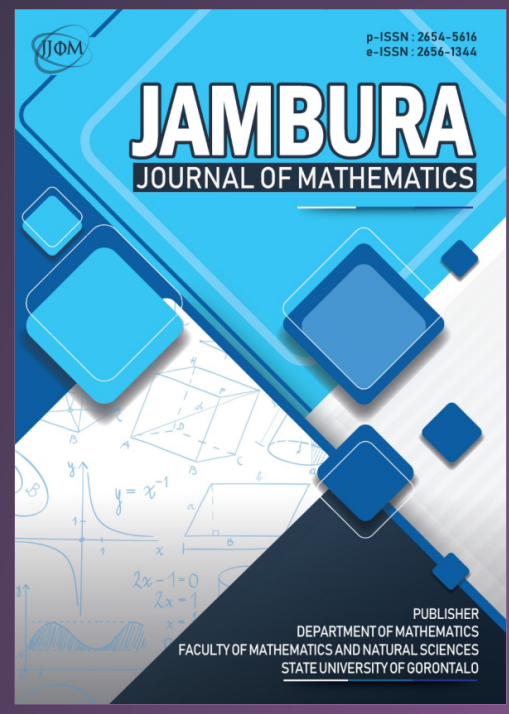

Jambura Journal of Mathematics

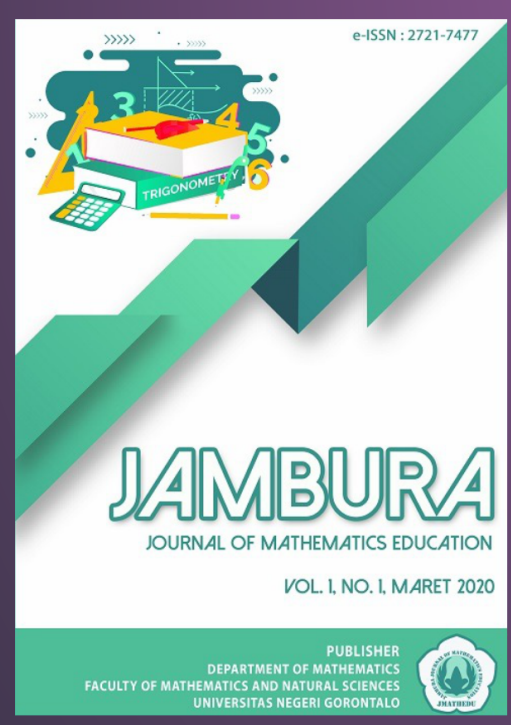

Jambura Journal of Mathematics Education
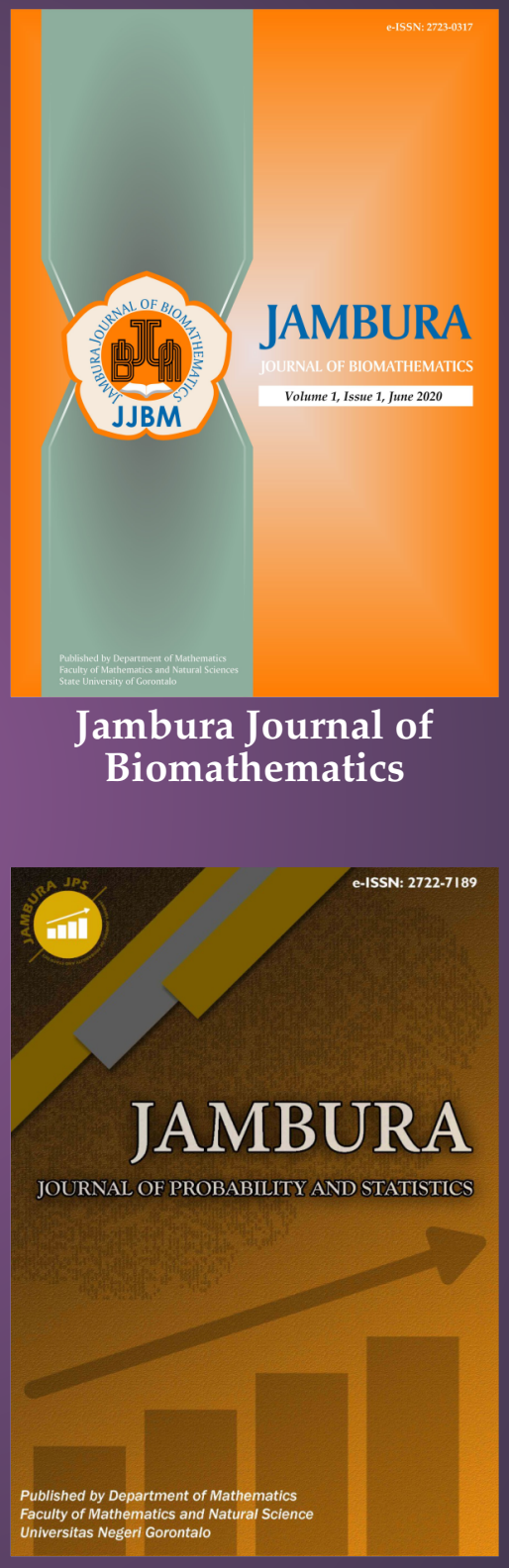

Jambura Journal of Probability and Statistics

Published by

Department of Mathematics

Faculty of Mathematics and Natural Sciences

State University of Gorontalo 